\title{
Statistical Analysis of High-Resolution Coherent Monopulse Radar Sea Clutter
}

\author{
Hong Zhu, Qingping Wang, Ning Tai, Jingjian Huang, and Naichang Yuan \\ College of Electronic Science and Engineering, National University of Defense Technology, Sanyi Avenue, Changsha 410073, China \\ Correspondence should be addressed to Hong Zhu; zhuhong13@nudt.edu.cn
}

Received 8 December 2016; Revised 10 March 2017; Accepted 14 March 2017; Published 11 May 2017

Academic Editor: Lorenzo Crocco

Copyright (C) 2017 Hong Zhu et al. This is an open access article distributed under the Creative Commons Attribution License, which permits unrestricted use, distribution, and reproduction in any medium, provided the original work is properly cited.

A statistical analysis that properly characterizes sea clutter processes is indispensable both for optimum detection algorithm design and for performance prediction problems in maritime surveillance applications. In this paper, we present the statistical analysis of experimental sea clutter data collected by a high-resolution coherent monopulse radar. First, we present the amplitude statistical analyses for these clutter data. The results show that the $K$, Pareto, and CIG distributions can each provide good fits to the clutter data for three channels of monopulse radar. The analyses on the variations of the $K$ distribution parameters with range suggest that the scale parameter is closely associated with the clutter powers and that the shape parameter is influenced by the sea state. Then, we focus on the correlation properties. The averaged results suggest that the temporal and spatial correlation properties are similar for the clutter of all three channels. Moreover, the clutter between the sum and difference channels is almost completely correlated in elevation and is lowly correlated in azimuth. Finally, we perform a spectral analysis, highlighting the temporal and spatial variabilities of Doppler spectra. It is found that the individual Doppler spectra in all three channels can be represented by Gaussian-shaped power spectral densities, and their centroid and width can be modeled as two separate stage linear functions of spectrum intensity.

\section{Introduction}

Maritime remote sensing and surveillance are of considerable importance. Radar has played a central role in these activities [1]. Monopulse, which uses the sum and difference beams to estimate the direction of arrival (DOA) of targets, is a mature angle measurement technique. Monopulse radars are widely used in target tracking systems because of their superior angular accuracy and powerful antijamming performance [2]. In recent years, combining the monopulse technology and the wideband imaging technology has become a general trend [3-6]. The wideband monopulse radars can synthesize one-dimensional high-resolution range profiles and twodimensional monopulse angle measurements to obtain threedimensional imaging of the target. Based on this threedimensional imaging, three-dimensional location information of scattering points of the target can be obtained. Furthermore, combining the two-dimensional SAR (synthetic aperture radar) or ISAR (inverse synthetic aperture radar) imaging, the target classification, feature detection, and the key part recognition of the target can be effectively achieved. Hence, the application of wideband monopulse radars can significantly improve the surveillance, the recognition, and the precise location of sea surface targets. When the wideband monopulse radar operates in a maritime environment, it will inevitably encounter sea clutter. This sea clutter is unwanted. It may interfere with the radar operation, irrespective of whether the wideband monopulse radar is used for detecting or tracking targets. A comprehensive understanding of the clutter environment in which radar operates is essential for evaluating the performance of maritime wideband monopulse radar. Moreover, to be able to optimize the signal processing under different conditions, the radar system designer also needs to have a detailed analysis of wideband monopulse radar clutter properties.

Due to the interaction of the sea surface characteristics, the geometry of acquisition, and radar parameters, there are apparent diversities of the statistical behaviors for the different clutter datasets recorded by different radar systems. Over the past few decades, various types of radar sea clutter 
datasets have been collected and studied [7-21]. The publications analyzing these datasets have primarily focused on suitable theoretical probability distribution function (PDF) models for sea clutter amplitude or intensity, the Doppler spectrum, the correlation characteristics, the sea spikes, and the average normalized radar cross section. These works can provide a thorough understanding of the statistical characteristics of sea clutter for other radar engineers when similar radar systems are applied to their designs. However, relatively little information has been published on monopulse radar sea clutter, particularly on high-resolution coherent monopulse radar sea clutter. This serves as the motivation to address this topic. To study the behavior of sea clutter received by a monopulse radar, we have constructed an experimental Ka-band wideband coherent monopulse radar system and conducted some pilot sea clutter trials.

A key aim of this study is to expand the knowledge of high-resolution coherent monopulse radar clutter through the detailed analysis of some experimental sea clutter datasets recorded by this wideband monopulse radar system, which has never been published in the previous literature. These datasets contain significant elements of novelty, which can help to provide more insights into the characteristics of monopulse radar sea clutter. It is well known that, in the conventional monopulse radar system, one common channel (sum channel) is used to transmit signals and three channels (sum channel and azimuth and elevation difference channels) are used to receive the clutter returns simultaneously. Although both the clutter returns are the backscatter from the same patch of ocean, the three receiver channels have different antenna patterns. Consequently, the recorded clutter data of the three receiver channels are neither exactly the same nor completely independent. There are some complex relationships among them. However, in the openly published literature, the corresponding analysis for this issue has still not been performed by applying the measured monopulse radar clutter. Therefore, the main element of novelty is the fact that the analysis presented within this work can provide a direct comparison of the clutter characteristics of three channels simultaneously to provide novel information on the difference and the relationship among the clutter of three channels of monopulse radar.

In this paper, the statistical analyses for these clutter data focus on three main properties: the amplitude statistics, the correlation characteristics, and the Doppler spectra analysis. Therefore, the statistical analysis techniques resemble previous woks in this field. The aim of the amplitude statistics is to obtain good models of the clutter amplitude by fitting different theoretical distribution models to the empirical distribution of the observed amplitude data of sea clutter. A variety of theoretical distribution functions have been proposed in the literature to model the amplitude probability density function of high-resolution sea clutter. Among them, we mention several commonly used PDFs: Weibull, $K$, Pareto, and compound inverse Gamma (CIG) [22-29]. As discussed in $[1,30,31]$, the parameters of these distribution models are influenced by the radar and environment parameters. Since the sea surface is highly nonstationary and the three channels of monopulse radar have different antenna patterns, when the same distribution is used to model the clutter of three channels, for three channels, the model parameters within the same range cell may be different, and, for each channel, they vary with range. To take into account the wide acceptance and application of the $K$ distribution model, studies about the variations of the $K$ distribution model parameter estimations with range are performed.

The correlation characteristics of sea clutter, which are particularly important for modeling and simulating clutter sequences and predicting detection performance involving temporal or spatial signal processing, include the temporal and spatial correlations [32-36]. In addition, many distribution models are special cases of the compound Gaussian model; thus, many simulations of sea clutter and target detection are performed on the assumption of the compound Gaussian model. Since the compound Gaussian model can be expressed as the product of texture and speckle components, the correlation function of clutter can also be written as the product of the correlation functions of the texture and speckle components. The texture and speckle components may exhibit different correlation characteristics due to their different physical mechanisms. In this paper, the correlation analyses are developed for these two components. Additionally, the clutter correlation property between the sum and difference channels may affect the angle measurement performance in the ordinary monopulse radar and the detection performance of moving targets in the monopulse-SAR (synthetic aperture radar). For the simulation of monopulse radar clutter, it should also be considered. Therefore, this correlation property is also investigated in this paper.

Modern radar systems can use coherent Doppler processing to suppress the noise and clutter interferences or to separate the targets from clutter in the Doppler domain. The characteristics of the Doppler spectra of sea clutter may have a significant effect on the results of the coherent Doppler processing. Understanding the characteristics of the Doppler spectra of sea clutter is critical for improvements in advanced signal processing algorithms. In $[37,38]$, the characteristics of the Doppler spectra of recorded sea clutter have been analyzed, and a simple method has been proposed for characterizing the temporal variation of the Doppler spectrum. To demonstrate the characterization and modeling of Doppler spectra of the recorded monopulse radar clutter, a similar analysis process is adopted here. Through investigating the key features of Doppler spectra (i.e., the shape of the Doppler power spectrum, the mean Doppler and width of individual spectra, and the average power of each spectrum), we want to extend the simple method to simultaneously characterize the temporal and spatial variabilities of Doppler spectra.

The remainder of this paper is organized as follows. In Section 2, the characteristics of the radar system and of the analyzed datasets are presented. Section 3 addresses the statistical characterization of the observed clutter amplitude by fitting different theoretical distribution models. The variations of the parameter estimations of the $K$ distribution model with range are investigated. The correlation analysis is presented in Section 4, which contains the temporal and spatial correlation analyses of each channel and the crosscorrelation analysis between the sum and difference channels. 


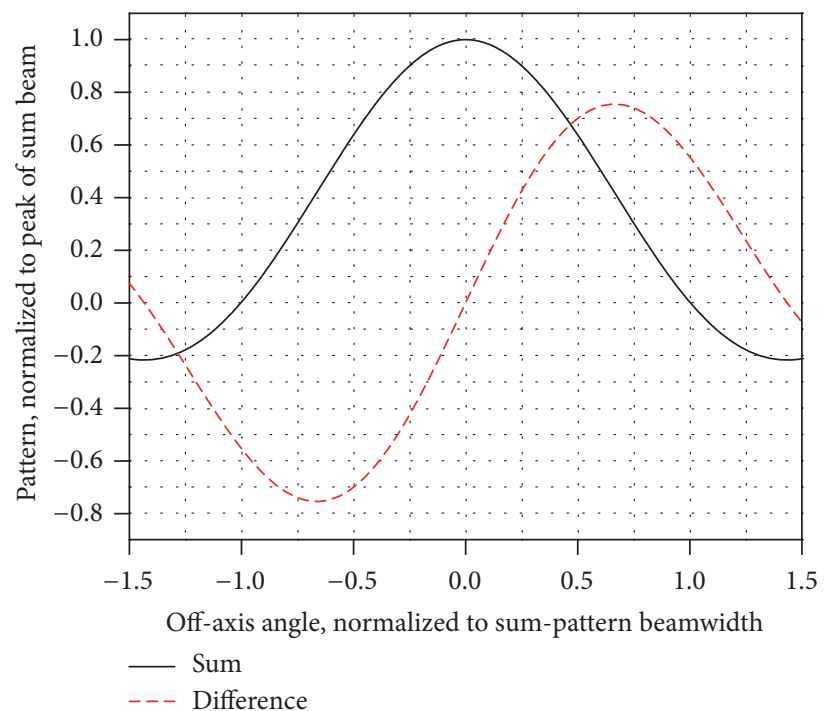

(a)

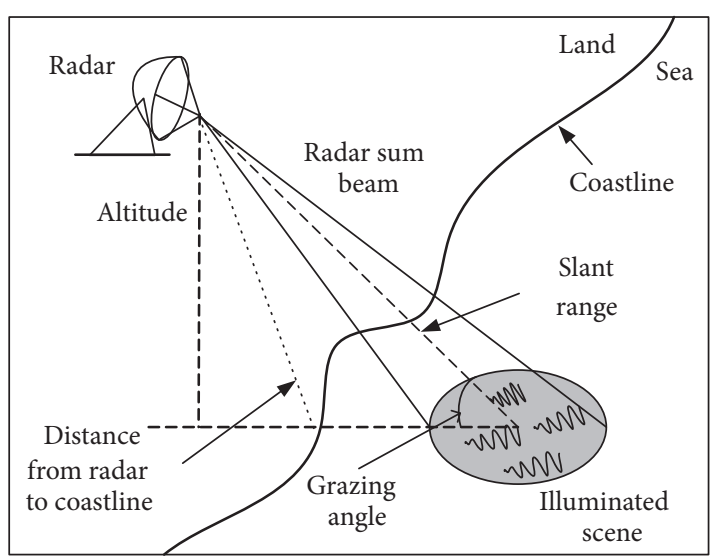

(b)

FIGURE 1: Sketches of the sum and difference patterns and the measurement geometry: (a) sketch of the sum and difference patterns; (b) sketch of the measurement geometry.

In Section 5, we analyze the Doppler spectra by investigating the key features of Doppler spectra. We explore a simple method for simultaneously characterizing the temporal and spatial variabilities of Doppler spectra. Finally, conclusions and discussions are presented in Section 6.

\section{Radar System and Data Description}

This section provides brief overviews of this wideband coherent monopulse radar system and the experimental setup. This radar system operates at the Ka-band and is coherent. It consists of a transmitter channel and three receiver channels: the sum channel and the elevation and azimuth difference channels. The three receiver channels can simultaneously record the clutter returns scattered from the same patches of ocean. The antenna patterns of the receiver sum channel and of the transmitter channel are the same, but those of the two receiver difference channels are different from that of the transmitter channel. Figure 1(a) presents a sketch of the sum and difference patterns for either elevation or azimuth. The sum pattern is a pencil beam with its peak on the boresight axis. For the two difference channels, the shapes of their receiving antenna patterns are similar, and they have their null on the boresight axis. Both the transmitter and receiver channels are designed as the vertical polarization. The radar transmits a train of chirp pulses, and its main characteristics are listed in Table 1. The signal bandwidth is $100 \mathrm{MHz}$, thus providing a theoretical range resolution of $1.5 \mathrm{~m}$. This radar system can work in two main modes: scanning and spotlight modes. When the radar is operating in spotlight mode, ISAR images of an unknown maneuvering marine target can be obtained continuously.

The sea clutter data analyzed in this paper were collected on 12 September 2013 in Huludao, Liaoning, China.
TABLE 1: Main characteristics of radar.

\begin{tabular}{lc}
\hline Property & Value \\
\hline Carrier frequency & $35.4 \mathrm{GHz}$ \\
Transmitted signal & Up linear chirp \\
Bandwidth & $100 \mathrm{MHz}$ \\
Pulse duration & Variable \\
Pulse repetition frequency & $500 \mathrm{~Hz}$ \\
Sampling frequency & $200 \mathrm{MHz}$ \\
Polarization (transmitter and receivers) & Vertical \\
Half-power beamwidth (Transmitter) & $3^{\circ}$ (el.) $\times 3^{\circ}$ (az.) \\
Tx. power & $15 \mathrm{~W}$ \\
\hline
\end{tabular}

Figure 1(b) shows the sketch of the measurement geometry. The radar operated in spotlight mode such that the same direction clutter data can be collected for a long time. The radar was set on a cliff $230 \mathrm{~m}$ above the sea. However, the distance between the radar and the coastline is over $800 \mathrm{~m}$. The radar only performed on the low grazing angle. During the measurement trials, two fixed grazing angles $\left(8^{\circ}\right.$ and $\left.10^{\circ}\right)$ were applied. To avoid confusion, the collected data under the two fixed low grazing angles are referred to as dataset 1 (DS1) and dataset 2 (DS2), respectively. In each file, the number of received samples per pulse (i.e., the number of range cells) is 4000. However, the received samples in many range cells are free of clutter due to the limitation of the antenna beamwidth. The considered subset of range cells are where the clutter is present. Here 1800 range cells for DS1 and 900 range cells for DS2 are selected to analyze. The number of time samples for each range cell is 31750 , which is equal to a recorded duration of 63.5 seconds. The main configurations of the analyzed datasets are summarized in Table 2. During the time the data 
TABLE 2: Main configurations of the analyzed datasets.

\begin{tabular}{lcccc}
\hline Dataset name & Grazing angle $(\mathrm{deg})$ & Slant range $(\mathrm{m})$ & Start range $(\mathrm{m})$ & Number of range samples \\
\hline DS1 & 8 & 1653 & 1140 & 1800 \\
DS2 & 10 & 1325 & 1042.5 & 900 \\
\hline
\end{tabular}

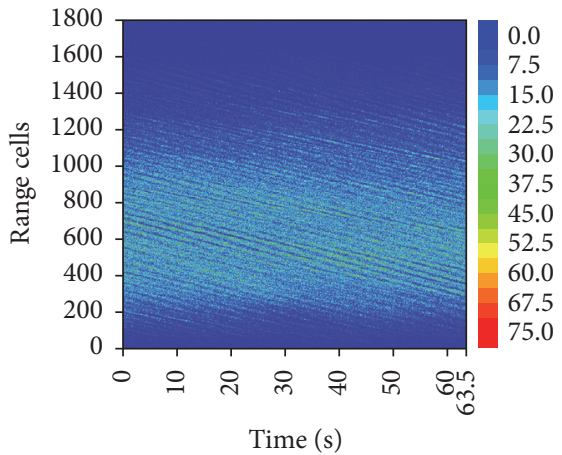

(a)

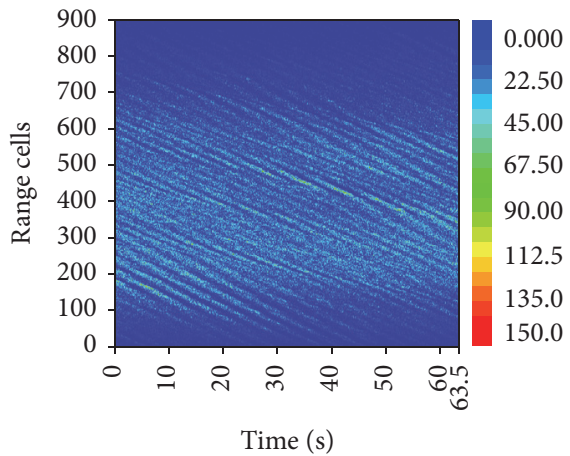

(d)

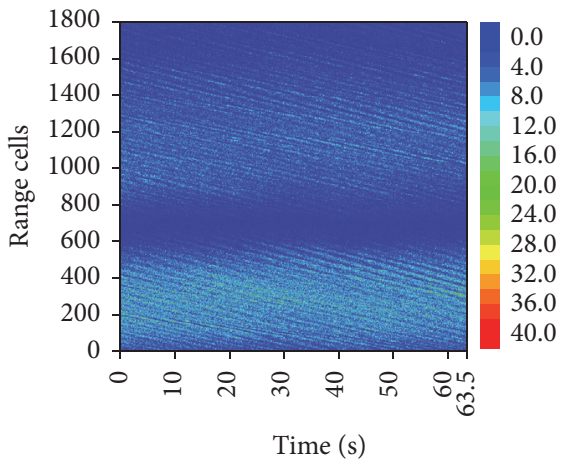

(b)

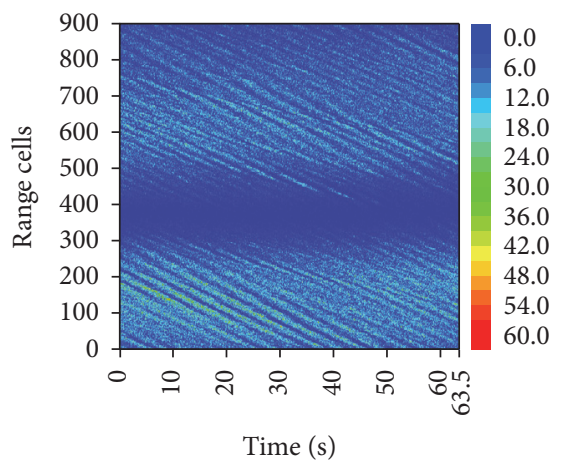

(e)

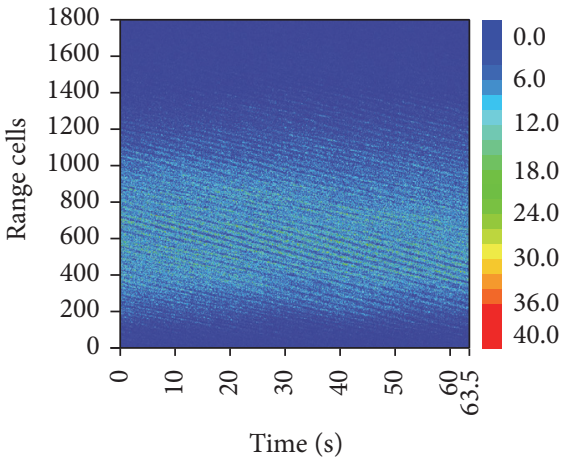

(c)

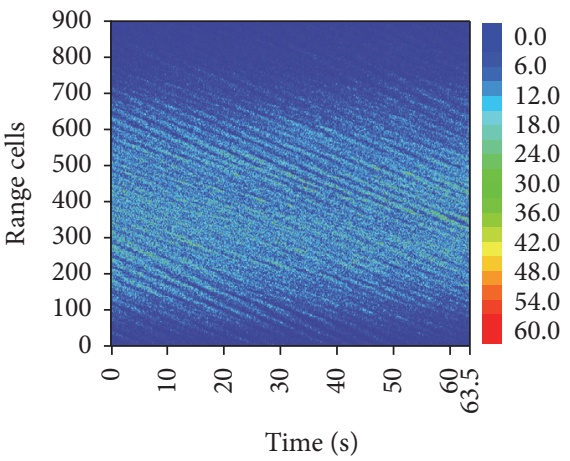

(f)

FIGURE 2: Sea clutter image in range against time format: ((a)-(c)) sum channel and elevation and azimuth difference channels for DS1. ((d)-(e)) Sum channel and elevation and azimuth difference channels for DS2.

were collected, the available wind recorded on the day showed a wind speed of approximately $9-10 \mathrm{~m} / \mathrm{s}$ blowing from the south direction. From the information about the wind speed, the sea state was approximately 4-5 (Beaufort scale). In the experiments, the available wave data on the day were not recorded. However, because of the waves close proximity to the shore, the waves were coming towards the coast line, and their direction can be approximately $315^{\circ}$ with respect to True North. The radar look direction was approximately $175^{\circ}$ with respect to True North. Therefore, the radar looks into the waves at an oblique angle of approximately $50^{\circ}$.

\section{Amplitude Analysis}

3.1. CNR. To provide a general view of the sea clutter data, amplitude-range-time (ART) maps of the recorded clutter samples have been obtained by considering the entire duration of the data. Figure 2 shows the ART maps of clutter samples in three channels for DS1 and DS2. In these maps, the horizontal axis corresponds to the time in seconds, the vertical one corresponds to the range cell number, and the color scale provides the amplitude. In each ART map, many bands of high amplitude can clearly be identified. This is because, in high-resolution radar systems, sea waves or swells can be resolved, and a great number of powerful scatterers can be located on the crest of the sea waves or swells. However, as stated by Ward et al. in [1] and by Palama et al. in [14], the Bragg scattering component, which is associated with resonant capillary waves, strongly influences the sea clutter returns at vertical polarization; thus, these ART maps clearly show the characteristic periodic variations of the underlying mean level of clutter with range and time due to the sea wave pattern. These wave-like modulations can contribute to the clutter "spikiness" despite not actually being composed of spikes [1]. They can contribute to the non-Gaussian nature of the clutter and have a significant effect on attempts of fitting empirical data to the theoretical distributions. This fact is further supported by the next results of the amplitude statistics analysis. 


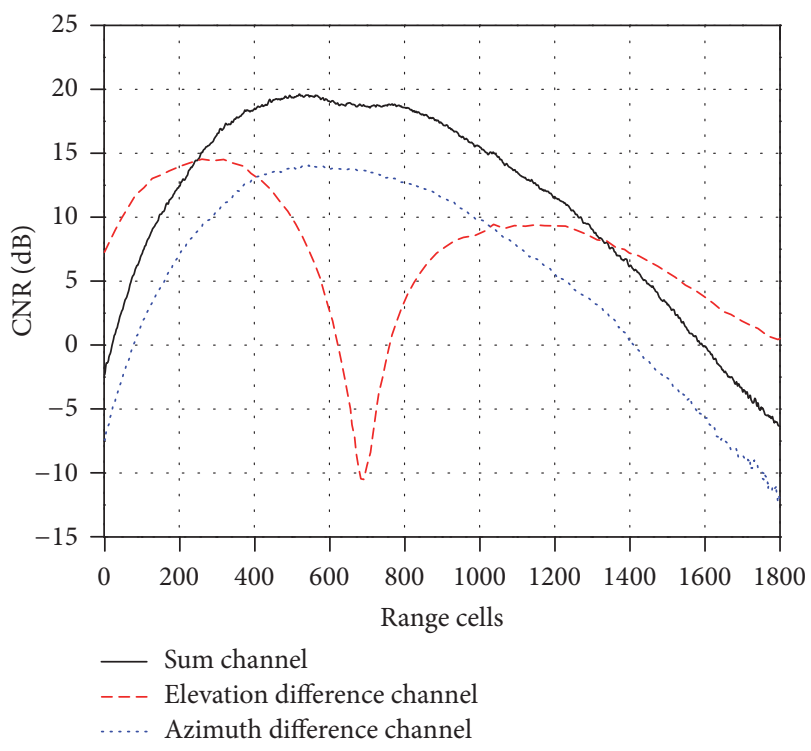

(a)

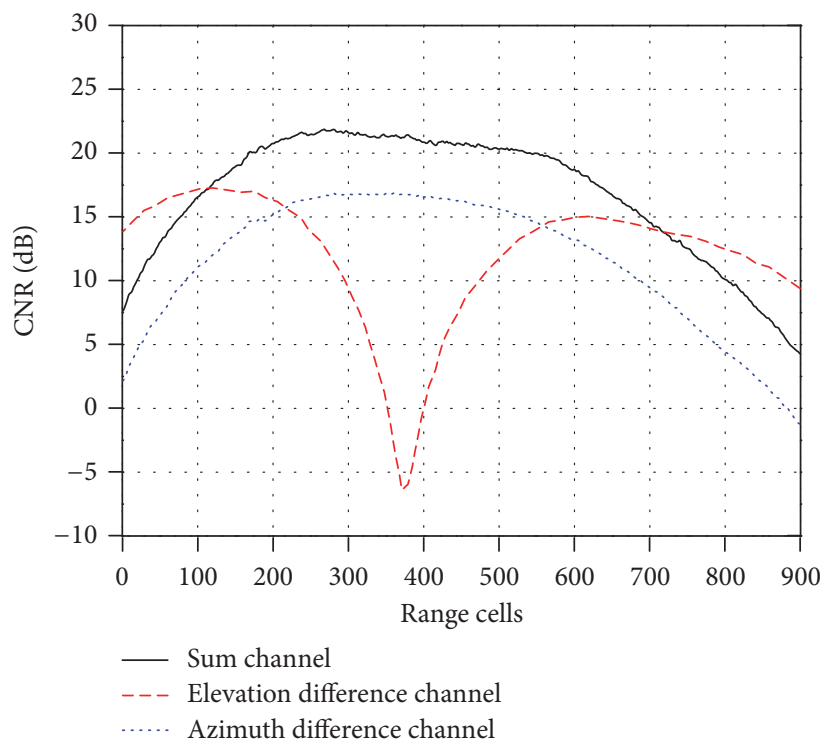

(b)

FIgURE 3: CNRs of the experimental sea clutter datasets: (a) DS1. (b) DS2.

For high-resolution radar systems, the corresponding illumination area of a single range cell is distributed over the entire region of the sum and difference patterns in the azimuth direction, but it is restricted within a small portion of the sum and difference patterns in the elevation direction. Consequently, in Figure 2, it can clearly be observed that the recorded sea clutter returns occur within a successive range cell region for both the sum and azimuth difference channels. For the elevation difference channel, however, the recorded sea clutter returns occur within two separate range cell regions.

For both sea clutter datasets, no specialized measurements were performed for the presence of only noise in clutter returns. However, a subset of range cells away from the clutter range cells can be carefully chosen to represent the background noise. The noise level is obtained by averaging all range cells and pulses of this subset. For each range cell, the average power of sea clutter plus noise is derived by averaging all powers over the full time record. The noise level is denoted as $p_{n}$, and the average power of clutter plus noise is denoted as $\langle z\rangle^{2}$. Then, the clutter to noise ratio (CNR) of each range cell can be estimated by

$$
\mathrm{CNR}=\frac{\langle z\rangle^{2}-p_{n}}{p_{n}} .
$$

Figure 3 shows the CNRs of the three channels for DS1 and DS2. In Figure 3, for both DS1 and DS2, the shapes of the CNR curves are similar between the azimuth difference and sum channels. They resemble the shape of the onedimensional sum antenna gain pattern shown in Figure 1(a). For all range cells, the CNRs of the azimuth difference channel are always lower than those of the sum channel. Compared with the CNR curves of the sum and azimuth difference channels, however, the CNR curves of the elevation difference channel are completely different. They have a deep slot. Their shape resembles the shape of the one-dimensional difference antenna gain pattern. Moreover, Figure 3 clearly shows that both DS1 and DS2 have two subsets of range cells in which the CNRs of the elevation difference channel are higher than those of the sum channel. However, all CNR curves are not severely symmetrical with respect to a center. This is because the clutter power falls off with increasing radar distance.

3.2. Statistical Models of Clutter Amplitude. The important step of the statistical analysis is to fit the probability density function (PDF) of the amplitude samples of the measured sea clutter by using the theoretical PDFs of some known distribution models. The Weibull, $K$, Pareto, and compound inverse Gamma (CIG) distributions are compared here. The analytical expressions of these PDFs $\left(p_{X}(x)\right)$ are reported as follows, where $x=|z(n)|$ is the amplitude of sea clutter and $z(n)$ is the complex envelope of clutter data.

(1) Weibull Model. The Weibull distribution model is commonly used in describing the non-Rayleigh clutter. Generally, the amplitude distribution of the high-resolution low-grazing-angle sea clutter can be accurately depicted by applying the Weibull model under the general sea condition $[22,23]$. Its PDF has the form

$$
p(x \mid a, b)=b a^{-b} x^{b-1} \exp \left(-\left(\frac{x}{a}\right)^{b}\right) u(x),
$$

where $u(x)$ is a step function, $a>0$ is a scale parameter, and $b>0$ is a shape parameter. The smaller the value of $b$ is, the higher the tails of the corresponding PDF are. Note that the exponential distribution and the Rayleigh distribution are particular cases of the Weibull distribution when the shape parameters $b=1$ and $b=2$. 
(2) $K$ Model. The compound $K$ distribution consists of two components of the envelope amplitude of the clutter returns $[1,24]$. The first is termed the speckle. The speckle is described as a complex Gaussian random variable; thus, its amplitude obeys the Rayleigh distribution. The second is termed the texture. The texture represents the local mean level and obeys the Gamma distribution. The speckle component is modulated by the texture component. The PDF of the $K$ distribution is given by

$$
\begin{aligned}
& p(x \mid \mu, v)=\int_{0}^{\infty} p(x \mid y) p(y) d y \\
& =\int_{0}^{\infty} \frac{\pi x}{2 y^{2}} \exp \left(-\frac{\pi x^{2}}{4 y^{2}}\right) \frac{2}{\Gamma(v)} b^{2 v} y^{2 v-1} \exp \left(-b^{2} y^{2}\right) \\
& \cdot u(x) d y=\frac{\sqrt{2 v}}{\sqrt{\mu} \Gamma(v) 2^{v-1}}\left(\sqrt{\frac{2 v}{\mu} x}\right)^{v} \\
& \cdot K_{v-1}\left(\sqrt{\frac{2 v}{\mu} x}\right) u(x),
\end{aligned}
$$

where $b=\sqrt{2 v / \mu \pi} \cdot \mu>0$ is the scale parameter, $v>0$ is the shape parameter, $\Gamma(\cdot)$ is a Gamma function, and $K_{v}(\cdot)$ is a second-order modified Bessel function of order $v$. Similar to the Weibull model, the smaller the shape parameter $v$ is, the higher the tails of the corresponding PDF are. The Rayleigh distribution is obtained for $v \rightarrow \infty$.

(3) Pareto Model. The Pareto distribution is a compound Gaussian model with a Rayleigh distribution being modulated by an inverse Gamma distribution [25-27]. Its PDF is given by

$$
\begin{aligned}
& p(x \mid \alpha, \beta)=\int_{0}^{\infty} \frac{2 x}{y} \exp \left(-\frac{x^{2}}{y}\right) \\
& \cdot \frac{\beta^{\alpha}}{\Gamma(\alpha)} y^{-(\alpha+1)} \exp \left(-\frac{\beta}{y}\right) u(x) d y=\frac{2 x \alpha \beta^{\alpha}}{\left(x^{2}+\beta\right)^{\alpha+1}} \\
& \cdot u(x),
\end{aligned}
$$

where $\alpha>0$ is the shape parameter and $\beta>0$ is the scale parameter. The power level of the Pareto-distributed random series will be higher with a larger scale parameter $\beta$. In addition, the smaller the shape parameter $\alpha$ is, the higher the tails of the corresponding PDF are.

(4) Compound Inverse Gaussian (CIG) Model. The compound inverse Gaussian model is a mixture of the Rayleigh distribution and the inverse Gaussian to model the speckle and the texture components, respectively [28]. In [29], experimental fitting results show that the sea clutter amplitudes obey the proposed CIG model in most cases. The PDF of the CIG distribution is given by

$$
\begin{gathered}
p(x \mid \lambda, \eta)=\int_{0}^{\infty} \frac{\pi x}{2 y^{2}} \exp \left(-\frac{\pi x^{2}}{4 y^{2}}\right) \\
\cdot \frac{\lambda^{1 / 2}}{\sqrt{2 \pi} y^{3 / 2}} \exp \left(-\lambda \frac{(y-\eta)^{2}}{2 \eta^{2} y}\right) u(x) d y,
\end{gathered}
$$

where $\eta>0$ is the mean and $\lambda>0$ is the shape parameter.

Since various theoretical distributions are involved, two common goodness-of-fit tests have been used to assess the suitability of each of these distributions for the measured sea clutter data.

(1) Root-Mean-Square Error (RMSE). Assume that $p(x \mid \widehat{\boldsymbol{\Theta}})$ is the theoretical distribution based on the estimated parameter vector $\widehat{\boldsymbol{\Theta}}$ and $\widehat{p}(x)$ is the estimated PDF from the real sea clutter data. Then, the RMSE for each distribution can be defined as

$$
\operatorname{RMSE}=\frac{1}{N} \sum_{k=1}^{N}(p(x(k) \mid \widehat{\boldsymbol{\Theta}})-\widehat{p}(x(k)))^{2},
$$

where $N$ is the sample length of the estimated PDF and $x(k)$ is the value of the sample.

(2) Chi-Squared (CS) Test. The theoretical CDF and the empirical CDF can be, respectively, expressed as $F_{X}(x(k))=$ $\sum_{i=1}^{k} p(x \mid \widehat{\boldsymbol{\Theta}}) d x$ and $\widehat{F}_{X}(x(k))=\sum_{i=1}^{k} \widehat{p}(x(k)) d x$, where $d x$ is the interval of two adjacent statistic samples. Thus, the CS test between the theoretical CDF $F_{X}(x(k))$ and the empirical $\operatorname{CDF} \widehat{F}_{X}(x(k))$ is defined as

$$
\mathrm{CS}=\sum_{k=1}^{N} \frac{\left(F_{X}(x(k))-\widehat{F}_{X}(x(k))\right)^{2}}{F_{X}(x(k))} .
$$

The smaller the CS test value, the better the fitting result of the theoretical distribution.

These distribution parameters are computed from the recorded data. The four most commonly used methods for estimating the parameters for these distribution functions have been proposed in the literature. The method of moments (MoM) (also known as moment matching) is to match the moments of the data sample to those of the distribution functions. This method is the simplest and fastest, but its accurate estimation requires very large data samples. As discussed in [39], the MoM is relatively inaccurate for the Pareto distribution clutter plus noise if the shape parameter is small. Maximum-likelihood estimators (MLEs) have been applied to Weibull [1], $K$ [40, 41], and Pareto [26] distributed clutter. These estimators can provide accurate results, but they are difficult to compute for clutter and noise because there is no closed form for the likelihood function. The $z \log (z)$ estimators have been applied to $K$ and Pareto distribution clutter $[1,39,42]$. When noise is included, these estimators still provide more robust and accurate estimates and are relatively quick to compute. There is no $z \log (z)$ estimator 
TABLE 3: Estimated parameters for different theoretical PDFs for DS1.

\begin{tabular}{|c|c|c|c|c|c|c|c|c|c|}
\hline \multirow{2}{*}{ Range cell } & \multirow{2}{*}{ Channel } & \multicolumn{2}{|c|}{ Weibull } & \multicolumn{2}{|c|}{ K } & \multicolumn{2}{|c|}{ Pareto } & \multicolumn{2}{|c|}{ CIG } \\
\hline & & $\widehat{a}$ & $\widehat{b}$ & $\widehat{v}$ & $\widehat{\mu}$ & $\widehat{\alpha}$ & $\widehat{\beta}$ & $\widehat{\eta}$ & $\widehat{\lambda}$ \\
\hline \multirow{3}{*}{ 200th } & Sum & 7.91 & 1.84 & 7.68 & 32.31 & 8.7 & 498.19 & 153.46 & 7.08 \\
\hline & Elevation & 7.79 & 1.86 & 9.26 & 31.17 & 9 & 501.78 & 158.76 & 6.99 \\
\hline & Azimuth & 4.3 & 1.89 & 12.38 & 9.41 & 17 & 298.06 & 197.85 & 3.8 \\
\hline \multirow{3}{*}{ 700th } & Sum & 15.81 & 1.83 & 5.98 & 130.2 & 7.14 & 1599.3 & 300.5 & 14 \\
\hline & Elevation & 1.66 & 1.99 & 395.56 & 1.37 & 30.54 & 82.77 & 126.76 & 1.48 \\
\hline & Azimuth & 8.49 & 1.85 & 7.86 & 37.36 & 7.57 & 499.2 & 202.46 & 7.57 \\
\hline \multirow{3}{*}{ 1200th } & Sum & 7.2 & 1.82 & 7.14 & 26.57 & 5.47 & 249.81 & 112.38 & 6.45 \\
\hline & Elevation & 4.76 & 1.84 & 8.93 & 11.46 & 10.58 & 218.98 & 148.91 & 4.18 \\
\hline & Azimuth & 3.79 & 1.91 & 15.43 & 7.19 & 18.4 & 249.38 & 200.08 & 3.37 \\
\hline
\end{tabular}

TABLE 4: Estimated parameters for different theoretical PDFs for DS2.

\begin{tabular}{|c|c|c|c|c|c|c|c|c|c|}
\hline \multirow{2}{*}{ Range cell } & \multirow{2}{*}{ Channel } & \multicolumn{2}{|c|}{ Weibull } & \multicolumn{2}{|c|}{$K$} & \multicolumn{2}{|c|}{ Pareto } & \multicolumn{2}{|c|}{ CIG } \\
\hline & & $\widehat{a}$ & $\widehat{b}$ & $\widehat{v}$ & $\widehat{\mu}$ & $\widehat{\alpha}$ & $\widehat{\beta}$ & $\hat{\eta}$ & $\hat{\lambda}$ \\
\hline \multirow{3}{*}{ 200th } & Sum & 20.11 & 1.79 & 4.36 & 217.38 & 5.08 & 1799.1 & 345.1 & 17.91 \\
\hline & Elevation & 10.16 & 1.78 & 4.77 & 55.16 & 7.5 & 697.63 & 151.8 & 9.12 \\
\hline & Azimuth & 10.17 & 1.84 & 7.55 & 52.91 & 8.54 & 799.46 & 246.51 & 8.99 \\
\hline \multirow{3}{*}{ 380th } & Sum & 21.34 & 1.71 & 3.38 & 259.56 & 3.25 & 1201.6 & 203.9 & 19.19 \\
\hline & Elevation & 1.76 & 2 & 49.64 & 1.56 & 39.63 & 120.69 & 155.97 & 1.57 \\
\hline & Azimuth & 12.05 & 1.84 & 8.67 & 74.07 & 6.14 & 799.65 & 253.63 & 10.74 \\
\hline \multirow{3}{*}{ 700th } & Sum & 10.03 & 1.85 & 7.12 & 52.1 & 7.62 & 696.77 & 247.93 & 8.89 \\
\hline & Elevation & 7.9 & 1.85 & 7.98 & 32.14 & 7.04 & 397.77 & 200.16 & 7.15 \\
\hline & Azimuth & 5.48 & 1.9 & 11.74 & 15.47 & 13.94 & 398.7 & 247.56 & 4.87 \\
\hline
\end{tabular}

available for the CIG distribution. The parametric curvefitting estimator (PCFE) ensures the estimation accuracy through minimizing the residuals between the experimental PDF, drawn from the recorded data intensity, and the fitted theoretical model PDF curves with unknown parameters $[43,44]$. This PCFE method can be applied to estimate the parameters for any sea clutter distribution.

In the high-resolution radar system, each range cell corresponds to a unique off-axis angle in elevation. According to the values of the corresponding off-axis angles in elevation, the entire range cells can be approximatively divided into one central region and two edge regions. As shown in Figure 3, in the central region, the elevation difference channel has a very low CNR, but the other two channels have high CNRs. In the other two edge regions, the CNRs of the three channels are not low, and the CNR differences between the sum and difference channels are not large in both azimuth and elevation. Therefore, in the experimental analysis, three different range cells have been chosen to be representative of the behavior for each range cell region for each dataset. In the fit processing, the MLE is used to estimate the parameters of the Weibull distribution, and the PCFE based on the N$M$ simplex method is used to estimate the parameters of the $K$, Pareto, and CIG distributions. In Tables 3, 4, 5, and 6, we report the optimal estimates of the parameters and the corresponding fitted errors (including the RMSE and the CS test) with regard to each theoretical distribution model for three channels' clutter for both DS1 and DS2.

As an example, in Figure 4, the assessments of the suitability of the different theoretical distributions to model the sea clutter amplitude PDFs of the three channels are depicted for the 200th and 380th range cells of DS2. To show how the theoretical model fits the tail of the empirical sea clutter amplitude distribution, the log 10 scale is used to plot the curves of the PDFs. The experimental results for the other range cells of DS2 and the other dataset are very similar. They are therefore not depicted here.

In Figure 4, the fitting curves show that, for the three channels and all range cells, the MLE can perform reasonably well in estimating the Weibull distribution parameters, and the PCFE can perform reasonably well in estimating the model parameters of both the $K$, Pareto, and CIG distributions. This behavior is better illustrated by the corresponding RMSE values, as illustrated in Table 6. In addition, visual inspection clearly shows that the $K$, Pareto, and CIG PDFs can perfectly match the tails of the empirical PDFs for the three channels and most of the range cells. This is also confirmed by the corresponding CS test values presented in Table 6. For the Weibull distribution, however, the good tail fitting of the empirical PDFs is obtained only in the case where the clutter data are from the 380th range cell in the elevation difference channel, as shown in Figure 4(e). In other 
TABLE 5: RMSE and CS test of the different theoretical PDFs for DS1 (in dB).

\begin{tabular}{|c|c|c|c|c|c|c|c|c|c|c|}
\hline \multirow{2}{*}{ Range cell } & & \multicolumn{3}{|c|}{ 200th } & \multicolumn{3}{|c|}{ 700th } & \multicolumn{3}{|c|}{ 1200th } \\
\hline & & Sum & Elevation & Azimuth & Sum & Elevation & Azimuth & Sum & Elevation & Azimuth \\
\hline CNR & & 13.03 & 12.87 & 7.12 & 19.22 & -9.67 & 13.73 & 12.2 & 8.23 & 5.73 \\
\hline \multirow{2}{*}{ Weibull } & RMSE & -53.36 & -53.16 & -47.85 & -57.73 & -39.17 & -53.02 & -50.60 & -46.54 & -47.23 \\
\hline & CS & -21.9 & -22.2 & -24 & -20.41 & -32.86 & -22.74 & -19.73 & -19.43 & -23.65 \\
\hline \multirow{2}{*}{$K$} & RMSE & -59.05 & -57.43 & -50.56 & -64.65 & -39.23 & -57.15 & -58.75 & -52.84 & -51.48 \\
\hline & CS & -34.3 & -34.14 & -31.43 & -34.46 & -32.19 & -33.61 & -33.38 & -27.88 & -29.52 \\
\hline \multirow{2}{*}{ Pareto } & RMSE & -58.61 & -57.29 & -50.31 & -63.13 & -39.02 & -57.25 & -57.87 & -52.71 & -51.48 \\
\hline & CS & -31.48 & -34.55 & -28.26 & -31.88 & -30.71 & -32.21 & -31.63 & -27.92 & -29.73 \\
\hline \multirow{2}{*}{ CIG } & RMSE & -58.03 & -55.83 & -50.57 & -64.4 & -38.73 & -57.2 & -56.16 & -52.92 & -51.57 \\
\hline & CS & -32.22 & -28.74 & -30.47 & -32.89 & -28.46 & -33.92 & -27.79 & -28.22 & -30.2 \\
\hline
\end{tabular}

TABLE 6: RMSE and CS test of the different theoretical PDFs for DS2 (in dB).

\begin{tabular}{|c|c|c|c|c|c|c|c|c|c|c|}
\hline \multirow{2}{*}{ Range cell } & & \multicolumn{3}{|c|}{ 200th } & \multicolumn{3}{|c|}{ 380th } & \multicolumn{3}{|c|}{ 700th } \\
\hline & & Sum & Elevation & Azimuth & Sum & Elevation & Azimuth & Sum & Elevation & Azimuth \\
\hline $\mathrm{CNR}$ & & 21.37 & 15.36 & 15.29 & 22.03 & -5.7 & 16.8 & 15.18 & 13.02 & 9.51 \\
\hline \multirow{2}{*}{ Weibull } & RMSE & -60.31 & -54.65 & -53.35 & -57.16 & -38.79 & -57.34 & -54.19 & -54.19 & -51.92 \\
\hline & CS & -20.2 & -21.4 & -20.12 & -16.4 & -32.7 & -21.97 & -20.87 & -21.1 & -27.34 \\
\hline \multirow{2}{*}{ K } & RMSE & -65.76 & -58.83 & -59.55 & -62.25 & -38.91 & -65.31 & -61.19 & -61.2 & -53.53 \\
\hline & CS & -33.84 & -32.72 & -31.04 & -30.96 & -33.2 & -33.43 & -30.88 & -32.22 & -33.1 \\
\hline \multirow{2}{*}{ Pareto } & RMSE & -63.21 & -55.8 & -59.46 & -66.57 & -38.89 & -63.8 & -60.85 & -60.76 & -53.32 \\
\hline & CS & -26.7 & -22.83 & -31.81 & -26 & -32.74 & -31.76 & -33.48 & -34.94 & -30.87 \\
\hline \multirow{2}{*}{ CIG } & RMSE & -63.67 & -58.36 & -59.77 & -64.49 & -38.78 & -63.94 & -61.1 & -61.09 & -53.42 \\
\hline & CS & -28.2 & -28.72 & -33.31 & -24.97 & -30.41 & -32.86 & -31.6 & -34.41 & -31.55 \\
\hline
\end{tabular}

cases, the Weibull distribution severely underestimates the tails of the empirical PDFs.

Since those theoretical distributions do not consider the thermal noise and the CNRs of DS2 are higher than those of DS1, the RMSEs of DS2 in Tables 5 and 6 are smaller than those of DS1. In addition, in Tables 3 and 4, the shape parameters of DS2 are smaller than those of DS1. This means that PDFs of DS2 have longer tails than that of DS1. The longer tails may bring the larger error between the theoretical CDF and the empirical CDF. Thus, the CS test values of DS2 in Tables 5 and 6 are larger than those of DS1. As expected, Tables 3 and 4 clearly show that, in the elevation difference channel, for both the 700th range cell of DS1 and the 380th range cell of DS2, the estimated Weibull distribution shape parameter is approximately equal to 2 . This means that the clutter data are now dominated by noise. The CNR values shown in Tables 5 and 6 illustrate this point directly. Moreover, in these cases, the values of the RMSE and the CS test also show that the fitted results of the Weibull distribution are almost similar to those of other reported distributions. Additionally, as shown in Tables 3 and 4, the parameters of each distribution vary from one range region to another range region for both DS1 and DS2. This is because the clutter backscattering itself is spatially heterogeneous and because the modulations of the clutter return amplitude by the transmitter and receiver antenna gain patterns vary with range in high-resolution radar. The results of Tables 5 and 6 show that the different distribution models produce different RMSE values for different range cells, and no model can always maintain the smallest fitted errors for all cases. Furthermore, the smallest values of RMSE and CS test are occasionally given by different PDF fits for the same clutter data. Nonetheless, the fitted errors of the $K$, the Pareto, and the CIG distributions are comparable and always quite small. Each of these distributions can be used as an accepted model for the three channels and all range cells.

3.3. K Distribution Parameters Analysis. According to the above analysis results, we can readily accept that the $K$ distribution model can be applied to effectively fit the amplitude statistical distribution of sea clutter data for the three channels of monopulse radar. Additionally, the $K$ distribution is widely used for analyzing the radar detection performance in sea clutter. There are two main benefits to investigating the variations of the $K$ distribution parameters with range for the high-resolution monopulse radar. On the one hand, some further information about the spikiness of clutter is provided by the value of the $K$ distribution shape parameter. On the other hand, the effects of the environmental and radar parameters on the radar sea clutter $K$ distribution shape parameter can be examined, and the relationship of $K$ distribution shape parameters of clutter among three channels can be obtained and generally understood. Such information can help the maritime radar designer to set 

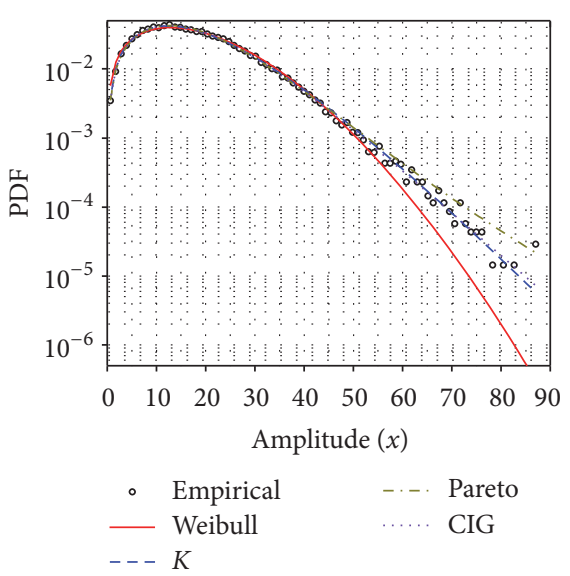

(a)

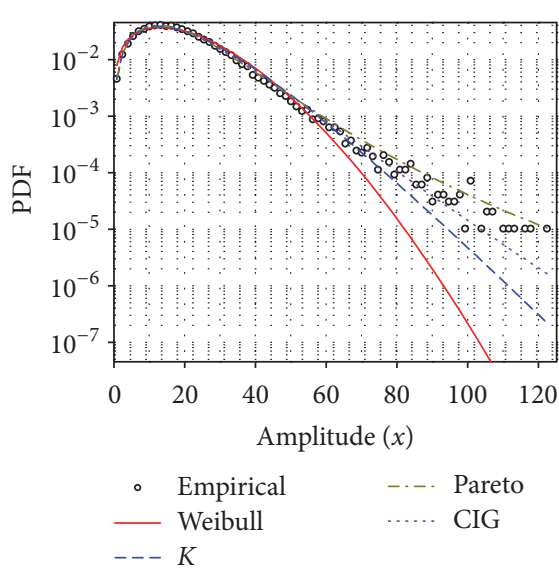

(d)

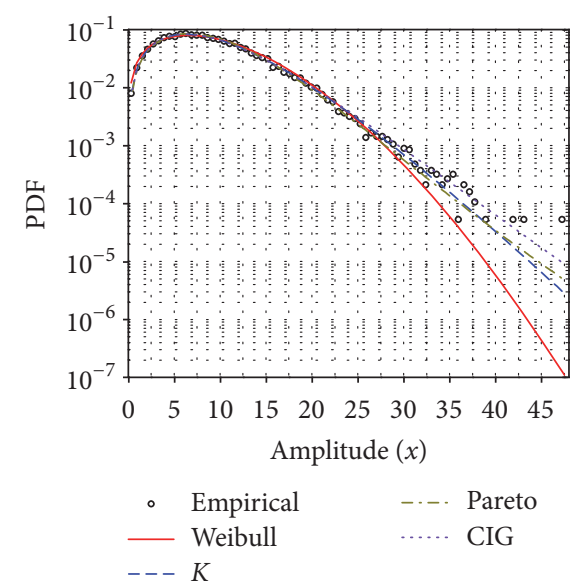

(b)

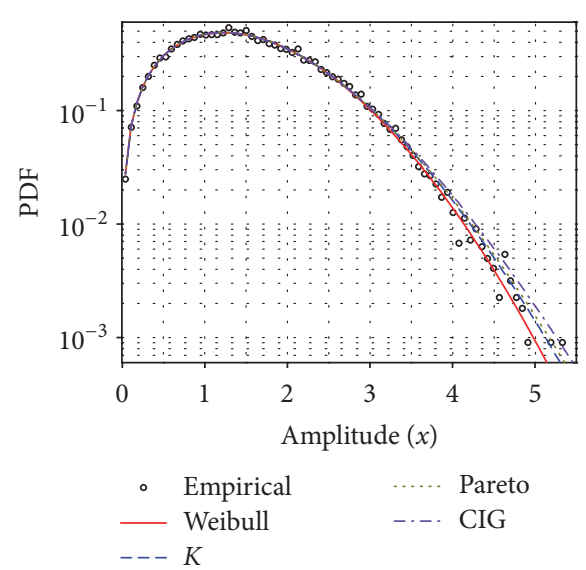

(e)

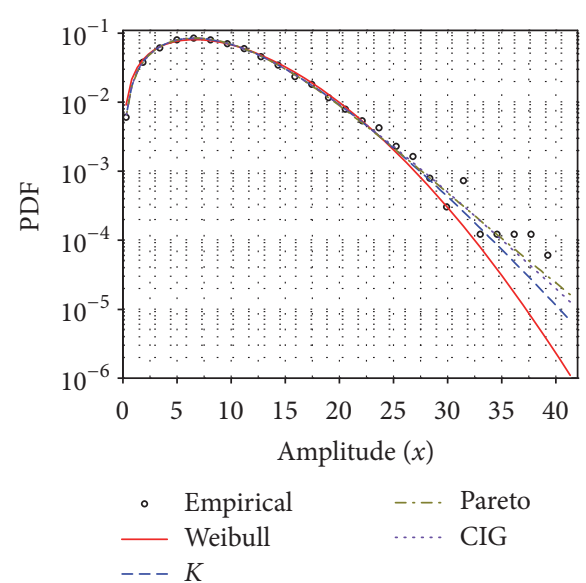

(c)

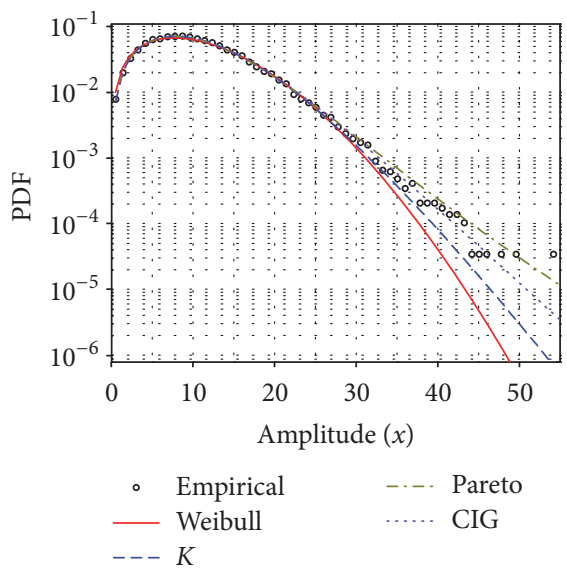

(f)

Figure 4: Examples of the fitted PDFs for DS2: ((a)-(c)) sum channel and the elevation and azimuth difference channels for the 200th range cell. ((d)-(e)) Sum channel and the elevation and azimuth difference channels for the 380th range cell.

reasonable $K$ distribution parameters before modeling $K$ distribution monopulse radar clutter.

Using the PDF given by (3), the second-order moment of the $K$ distribution can be represented as $E\left\{X^{2}\right\}=2 \mu$. This equation implies that the scale parameter of the $K$ distribution is determined by the clutter power. Consequently, the same information of the $K$ distribution scale parameter is contained in the CNR plots in Figure 3, and its plots do not need to be depicted here.

For DS1 and DS2, the fitted results of the $K$ distribution shape parameters in the three channels are shown in Figure 5, where the thermal noise is ignored. The plots are obtained by averaging the estimated parameters over a sliding range cell segment. There are 10 range cells per segment. A 50\% overlap between adjacent segments has been used. For each range cell, the $K$ distribution parameters are estimated by the PCFE. For DS1, the results of the azimuth difference channel in the region from the 1601st to 1800th range cells are unacceptable. The results of the three channels in this range cell region are hence discarded.

In Figure 5, it can be observed that the estimates of the shape parameter of the elevation difference and sum channels are close in those range cells where the clutter powers of both the elevation difference and sum channels are higher, and they are smaller than those of the azimuth difference channel, meaning that the clutter returns in the sum and elevation difference channels have longer tails than those in the azimuth difference channel. In addition, as mentioned previously, the noise alone would provide an effective value of $v=\infty$. From Figure 3, we can see that three channels of DS1 at the near and far range cells, azimuth difference channel of DS2 at far range cells, and elevation difference channel of DS1 and DS2 at middle range cells have low CNR. Thus, it is evidently shown in Figure 5 that the $K$ distribution shape parameters at those low CNR range cells are much greater.

However, in a real environment, the data are generally corrupted with the thermal noise. To correct the effect of thermal noise, we again estimate the $K$ distribution shape parameter by taking into account the presence of thermal noise. Although in this case the PCFE can achieve good estimation performance of the shape parameter of the $K$ distribution, it is computationally expensive. As discussed in $[1,16]$, in the usual situation where the noise level is known, the method to estimate the shape parameter of the 


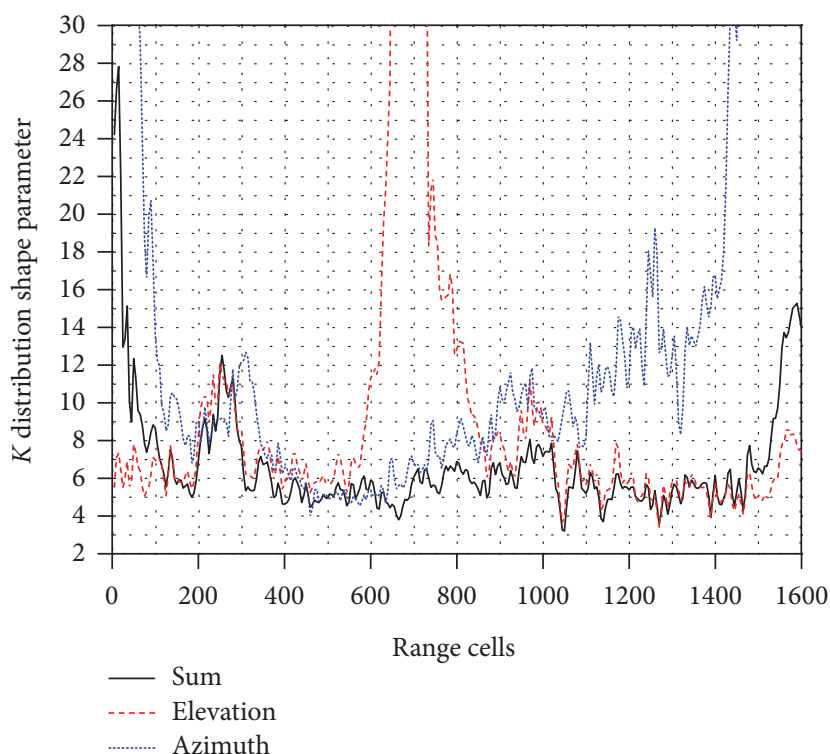

(a)

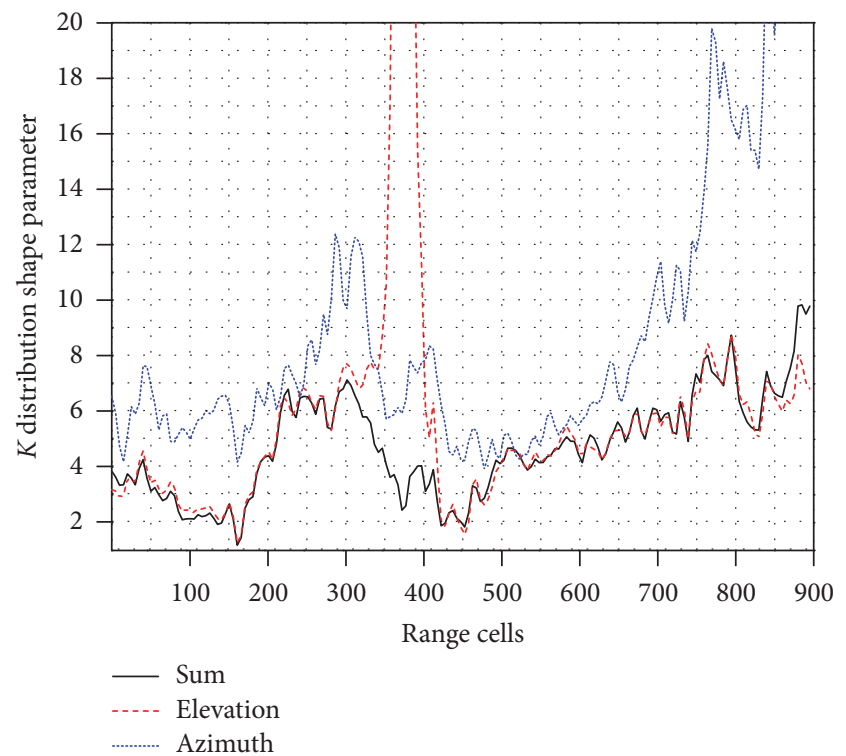

(b)

FIGURE 5: Estimated $K$ distribution parameters, where the thermal noise is ignored: (a) shape parameter for DS1. (b) Shape parameter for DS2.

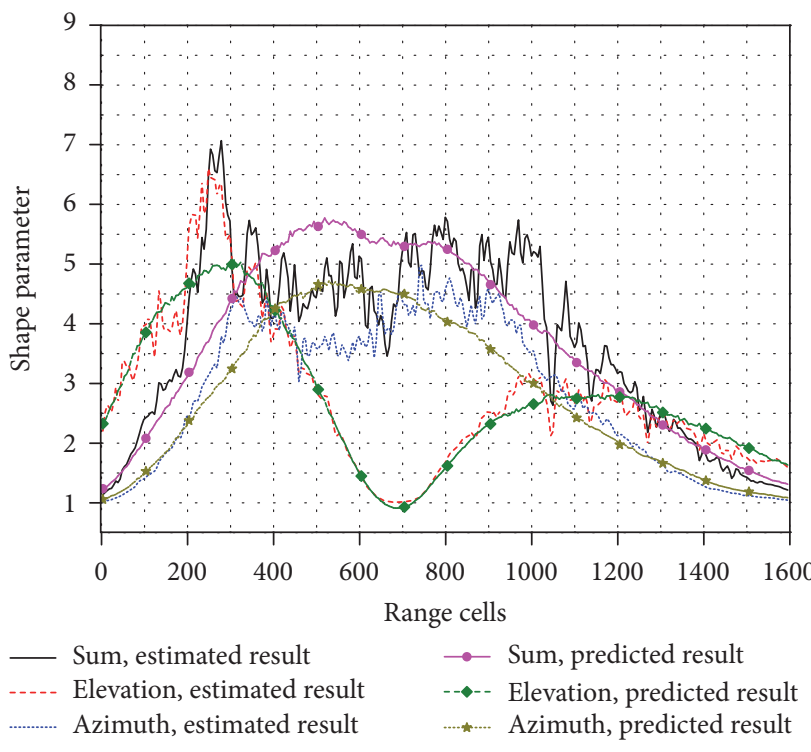

(a)

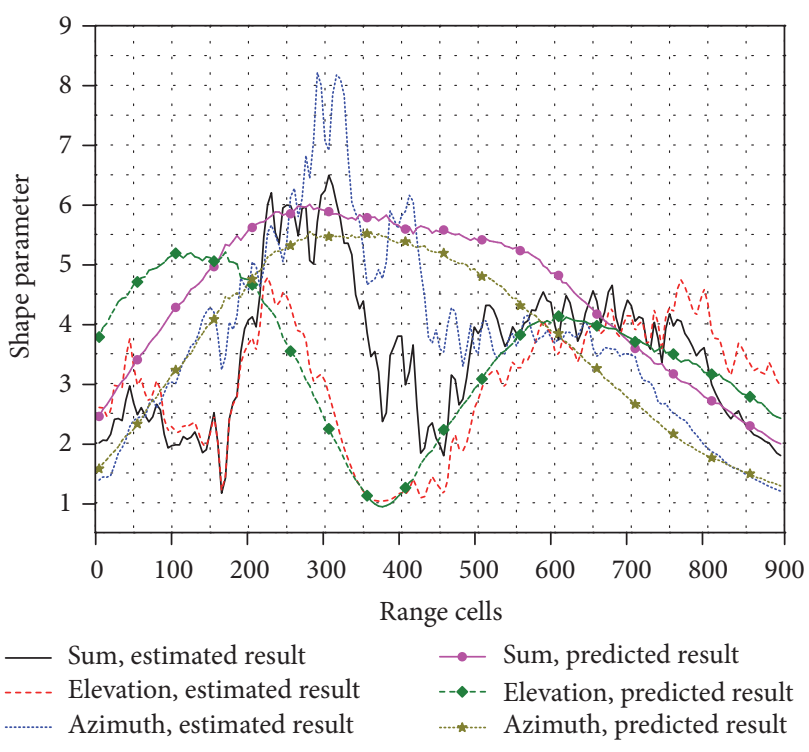

(b)

Figure 6: Estimated and fitted $K$ distribution shape parameter, where the thermal noise is considered: (a) DS1. (b) DS2.

$K$ distribution by using the noise power estimate and higherorder moments can not only ensure the estimation accuracy but also improve the estimation speed. Therefore, this MoM based on the estimated noise power is used here to estimate the shape parameter. The results of the estimated shape parameter are depicted in Figure 6.

If $\widehat{v}$ is the shape parameter of $K$ distribution clutter in the absence of thermal noise and if $\widehat{v}_{\text {eff }}$ is the equivalent estimate when thermal noise is present, then we have $\widehat{v}=$ $\widehat{v}_{\text {eff }}\left(1+\mathrm{CNR}^{-1}\right)^{-2}[1]$. Accordingly, for those range cells where
CNR is high, the effect of noise on the shape parameter estimate is minimal; thus, as shown in Figures 5 and 6, the values of the shape parameters for $K$ distributions in the two cases are consistent. For those range cells where CNR is low, the values of the $K$ distribution shape parameters in the two cases are not consistent: the value of $\widehat{v}_{\text {eff }}$ is large, but the value of $\widehat{v}$ is still small, which means that the unwanted effects of thermal noise are very effectively removed from our estimates of $\hat{v}$. Notably, for the two cases, the values of the $K$ distribution shape parameters are both lower in those range 
cells where the severe banded patterns are present in the ART maps shown in Figure 2. This means that the sea clutter is spikier in those range cells. As stated by Ward et al. in [1], the backscatter in our trials is predominantly due to Bragg scattering from tilted, slightly rough surfaces and scattering from whitecaps (which appears to arise from the very rough surfaces of waves as they break). In our results, the presence of spikes can be associated with the whitecap component. Again, the plots shown in Figures 2, 5, and 6 suggest a possible relation between the presence of bands of high power and the occurrence of long-lasting spikes (whose duration is included between $1 \mathrm{~s}$ and approximately $3 \mathrm{~s}$ ) $[1,14]$.

An empirical model for the shape parameter of the $K$ distribution was proposed in $[1,30,31]$ :

$$
\log _{10}(v)=B \log _{10}\left(\phi_{\mathrm{gr}}\right)+C \log _{10}\left(A_{c}\right)+D,
$$

where $\phi_{\mathrm{gr}}$ is the grazing angle in degrees, $A_{c}$ is the area of the clutter-illuminated patch, and $B, C$, and $D$ are the constant parameters. In our monopulse radar system, the beam width of the sum antenna pattern is narrow and the grazing angle is approximately $8^{\circ}$ for DS1 and approximately $10^{\circ}$ for DS2. According to this empirical model, for different range cells, the effect of the changing grazing angle on the $K$ distribution clutter shape parameter is small and can be ignored. The variation of the shape parameter is now primarily dominated by the effective area of the clutter-illuminated patch of each channel, which is determined jointly by the transmitter and receiver antenna patterns and can be associated with the CNR. Thus, this model can be simply expressed as $\log _{10}(v) \approx$ $C^{\prime} \log _{10}(\mathrm{CNR})+E$, where $C^{\prime}$ and $E$ are constant parameters and assumed to have the same dependency of the radar and environment parameters for all range cells of each channel.

In Figure 6, the shape parameter predicted by applying this simple empirical model is also shown. As shown, the estimated results exhibit similar variation trends with the predicted results in most range cells of DS1 and some range cells of DS2. In other range cells, they appear to be completely divergent. For example, in Figure 6(b), the values of the estimated shape parameters for both the sum and elevation difference channels are lower than those for the azimuth difference channel in the region from the 100th to 200th range cells. However, the predicted results show that they are higher. In addition, in the region of DS2 (from the 50th to 150th range cells), the values of the predicted shape parameters increase with the increases of the range cells, but the values of the estimated shape parameters do not increase for both the elevation difference and sum channels. This may be caused by the occurrence of long-lasting spikes, which can be associated with the presence of the severe banded patterns (which are originated from the returns of the crest of the sea waves probably when they are about to break) in the ART maps shown in Figure 2 [14]. Consequently, this suggests that the variation of the shape parameter is dependent not only on the effective illumination area but also on the sea conditions, as discussed by Watts et al. in [31]. Due to a shortage of data, further analysis of the effects of the sea conditions on the shape parameter of the $K$ distribution cannot be performed in this paper. Nonetheless, note that, in Figure 6(a), for each channel, the overall shape of the curve of the estimated shape parameter exhibits an approximately similar trend with that of the predicted shape parameter. This result implies that, in the homogeneous sea surface, for each range cell, the $K$ distribution clutter shape parameters of the three channels of wideband monopulse radar may be determined by their respective illumination sea area when the thermal noise is considered.

Note that the sea-spike component, which describes the strong and rapidly fluctuating events, causes the commonly used $K$ distribution model to break down, particularly at finer resolutions where they result in higher levels of backscatter and extend the tail of the distribution. The $K A$ distribution is developed as an extension of the compound $K$ distribution to include the effects of spiking events by modeling them as discrete scatterers [45]. In [13], the $K A$ distribution was reported to provide the best fit for most of the datasets by a comparative analysis of fitting five different distributions to bistatic clutter data. However, it is perceived as being computationally expensive to use in practice. Dong therefore introduced the $K K$ distribution to capture the high-valued backscatter associated with the sea-spike component using two discrete $K$ distributions [46]. Further analyses of the fits of the $K A$ and $K K$ distributions to those datasets and of the variation of their shape parameters with range go beyond the scope of this paper and will be covered in future work.

\section{Correlation Analysis}

For the coherent radar system with a fine resolution, the received sea clutter returns are correlated. The correlated characteristics of the sea clutter include the temporal and spatial correlations. The temporal correlation of sea clutter refers to the correlation of multiple pulse returns within the same range cell. The spatial correlation of sea clutter refers to the correlation of multiple range cells returns from the same pulse.

According to the compound Gaussian model, the discrete-range-time expression of the complex envelope of high-resolution sea clutter can be described as the product of two independent components; that is,

$$
\begin{aligned}
z(r, n)=s(r, n) g(r, n) & =\sqrt{\tau(r, n)} g(r, n), \\
r & =1,2, \ldots, R, n=1,2, \ldots, N,
\end{aligned}
$$

where $R$ and $N$ are the numbers of discrete samples along range and time, respectively. Factor $\tau(r, n)$ is the texture, which is a slowly varying nonnegative real random process. Factor $g(r, n)=g_{I}(r, n)+j g_{\mathrm{Q}}(r, n)$ is the speckle, which is a stationary zero mean complex Gaussian process; $g_{I}(r, n)$ and $g_{Q}(r, n)$ are the in-phase $(I)$ and quadrature $(Q)$ components of $g(r, n)$ and share the same variance. They satisfy $E\left\{g_{I}(r, n)\right\}=E\left\{g_{Q}(r, n)\right\}=0$, with $E\left\{g_{I}^{2}(r, n)\right\}=$ $E\left\{g_{Q}^{2}(r, n)\right\}=1 / 2$ and thus $\left\{g^{2}(r, n)\right\}=1 . E\{\cdot\}$ is the expectation operator. 
According to (9), the overall autocorrelation function of sea clutter can be written as the product of two correlation functions $[10,47]$

$$
R_{z}(m)=E\left\{z^{*}(l+m) z(l)\right\}=R_{\tau}(m) R_{g}(m),
$$

where $R_{g}(m)$ and $R_{\tau}(m)$ are the autocorrelation functions for the speckle and texture components, respectively. Note that the speckle correlation $R_{g}(m)$ consists of multiple terms, that is, the autocorrelation of the in-phase and quadrature speckle components (real part) and the cross-correlation between them (imaginary part). The lag number $m$ can be in either the range or time direction. For instance, if the lag number $m$ is in the time direction, $z(l)$ and $z(m+l)$ refer to sea clutter data collected in the $l$ th and $(m+l)$ th pulses from the same range cell and $R_{z}(m), R_{\tau}(m)$, and $R_{g}(m)$ now refer to the temporal correlations. However, if the lag number $m$ is in the range direction, $z(l)$ and $z(m+l)$ refer to sea clutter data collected in the $l$ th and $(m+l)$ th range cells from the same pulse and $R_{z}(m), R_{\tau}(m)$, and $R_{g}(m)$ now refer to the spatial correlations. Equation (10) clearly shows that the correlation of sea clutter is influenced by the correlation characteristics of the speckle and texture components. Due to their different physical origins, the speckle and texture components present very different correlation lengths for both the temporal and spatial correlations.

4.1. Estimation of Texture Sequences. The texture $\tau(r, n)$ can be considered as constant within a proper time slot, which is defined as the coherence time of the texture. $\Delta T$ denotes the radar pulse repetition interval, and $L_{c}$ denotes the coherence length of the texture; thus, the coherence time of the texture can be denoted as $\Delta T L_{c}$. In practice, $\Delta T L_{c}$ satisfies that $\Delta T L_{g} \ll \Delta T L_{c} \ll \Delta T L_{s}$, where $\Delta T L_{g}$ and $\Delta T L_{s}$ are the speckle and texture correlation times, respectively. During each $L_{c}$ sample time, $z(r, n)$ can be represented as the product of a random constant times a Gaussian process. Assuming that the thermal noise is negligible, the texture sequence $\tau(r, l)$ can be then estimated using a moving-window (MW) filter of length $L_{c}$ as [47-50]

$$
\begin{aligned}
& \widehat{\tau}(r, l)=\frac{1}{L_{c}} \sum_{n=1+(l-1) L_{c} / 2}^{(l+1) L_{c} / 2}|z(r, n)|^{2} \\
& \cong \tau(r, l) \frac{1}{L_{c}} \sum_{n=1+(l-1) L_{c} / 2}^{(l+1) L_{c} / 2}|g(r, n)|^{2}, \\
& \quad l=1,2, \ldots, N_{b},
\end{aligned}
$$

where $N_{b}$ is the number of bursts in which the entire time sequence of each range cell data has been divided. Note that, with $50 \%$ of overlap, we obtain the estimation of texture sequence only every $L_{c} / 2$ steps. Through the approach of one-dimensional interpolation, the approximate estimation of texture sequence $\widehat{\tau}_{a}(r, n)$ for all discrete time samples can be obtained. The hypothetical speckle time sequence is then estimated as

$$
\widehat{g}(r, n)=\frac{z(r, n)}{\sqrt{\widehat{\tau}_{a}(r, n)}} .
$$

To estimate the sea texture and speckle sequences, the coherence length of the texture needs to be measured first for each dataset. In this paper, the coherence length of the texture can be estimated by employing a similar procedure based on the Jarque-Bera test, as given in [11]. However, in our procedure, the real and imaginary parts of $\widehat{g}\left[r, n ; \widehat{L}_{c}\right]$ are used together to perform a Gaussian statistical test on the null hypotheses, where $\widehat{g}\left[r, n ; \widehat{L}_{c}\right]$ is the estimated complex speckle sequence for the hypotheses coherence length $\widehat{L}_{c}$.

The estimated coherence length of the texture for DS1 is 200 and that for DS2 is 160 . Therefore, MW filters with coherence lengths of 200 samples and of 160 samples are, respectively, used to estimate the clutter texture sequences of all range cells in the three channels for DS1 and DS2. A 50\% overlap between two adjacent MW filters is used. Figure 7 shows the range-time maps for the estimated texture results. It can be clearly observed from Figure 7 that the banded patterns of high magnitude also occur in all range-time maps of the estimated texture. In the compound Gaussian model, the texture component describes the underlying power of the data and is associated with long waves and swells. Due to the periodic structure of the sea waves and swells, the estimated textures exhibit periodic behavior with range and time in a similar manner to the data in Figure 2. As documented theoretically by Conte and Longo in [51] and empirically by Haykin and Thomson in [52], it is an acceptable assumption that the sea clutter is cyclostationary in the three channels for both DS1 and DS2. The texture component of the clutter can therefore be modeled as a sum of real cosinusoid terms with unknown frequencies, amplitudes, and phases. The detailed estimation methods for this cyclostationary texture model and its model parameters have been proposed by Gini and Greco in [48] and by Ing et al. in [49].

4.2. Estimation of the Temporal Correlation Functions of Sea Clutter. The speckle component is often described as resulting from a uniform field of many random scatterers of the clutter in any range cell. After removing the texture component from sea clutter data, the estimated speckle can be assumed to be homogeneous. Using the estimated speckle results, the temporal autocorrelation function for the speckle can be estimated by employing the following estimator with $N_{g}=256$ data samples and $M_{g}=100$ data blocks:

$$
\begin{aligned}
& R_{t, g}(m)=\frac{1}{M_{g}} \sum_{n=1}^{M_{a}}\left[\frac{1}{N_{g}}\right. \\
& \left.\quad \cdot \sum_{l=1+(n-1) N_{a} / 2}^{(n+1) N_{a} / 2-m}\left[\hat{g}^{*}(r, l+m)-\bar{g}_{n}^{*}\right]\left[\hat{g}(r, l)-\bar{g}_{n}\right]\right],
\end{aligned}
$$

where $\left|\bar{g}_{n}\right|=\left(1 / N_{g}\right) \sum_{l=1+(n-1) N_{a} / 2}^{(n+1) N_{a} / 2} \widehat{g}(r, l)$ is the statistical expectation of $N_{g}$ time samples of the estimated complex speckle within the same range cell.

Figure 8 shows the results of this correlation analysis for DS2. In Figure 8, the real and the imaginary parts of the temporal correlation coefficients $\left(\rho_{t, g}=R_{t, g}(m) / R_{t, g}(0)\right)$ 


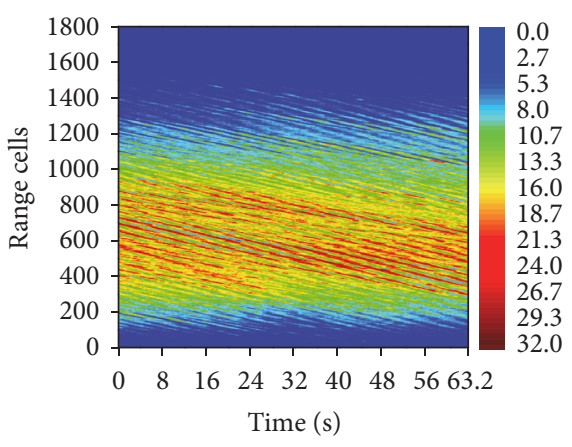

(a)

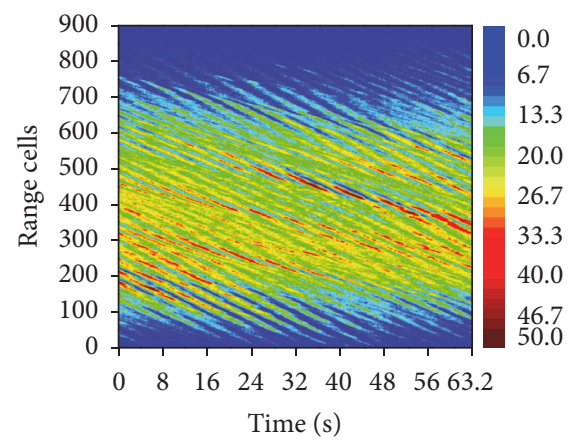

(d)

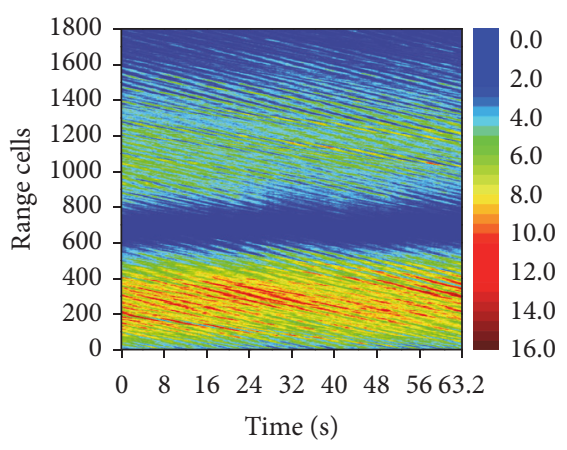

(b)

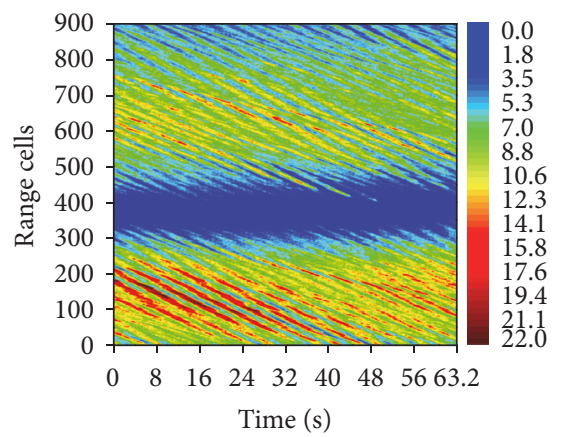

(e)

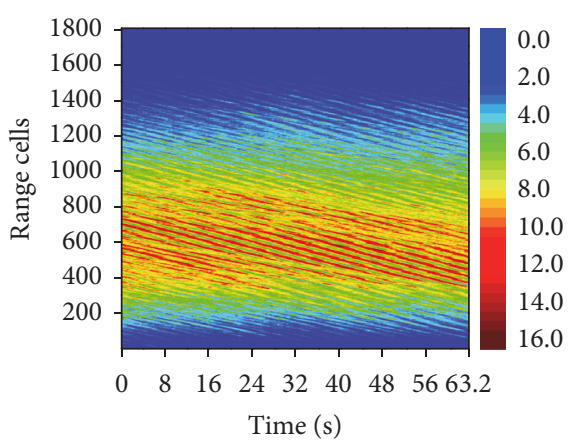

(c)

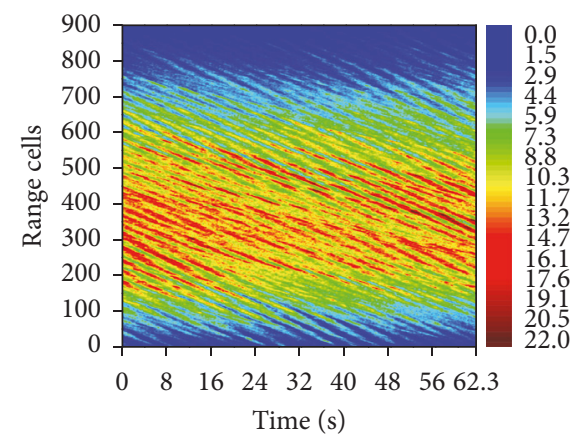

(f)

FIGURE 7: The images of estimated texture components in range against time format: (a) sum channel and elevation and azimuth difference channels for DS1. ((d)-(f)) Sum channel and elevation and azimuth difference channels for DS2.

of the speckle are plotted. Two representative range cells (e.g., the 200th and 380th range cells) are carefully chosen to illustrate the different behaviors of the three channels for the entire dataset. In the 200th range cell, the three channels of both have middle clutter intensity. The temporal correlation coefficient estimations of the speckle for the three channels do not show apparent differences. In the 380th range cell, the azimuth difference and sum channels have high clutter intensity, but the elevation difference channel has low clutter intensity. Thus, the temporal correlation coefficient estimations of the speckle for the elevation difference channel are lower than those for both the azimuth difference and sum channels. Additionally, for the estimations in both the azimuth difference and sum channels, there are also no apparent differences between the 200th and 380th range cells. Due to the rapidly varying nature of the speckle component, the correlation time of the speckle component is around milliseconds, and here, the overall results indicate that it is approximately $7 \mathrm{~ms}$ for all channels in the range cells of middle and high clutter intensity. Furthermore, the imaginary parts of the temporal correlation coefficient of the speckle components are nonnegligible. These terms are closely associated with the Doppler frequency shift of the clutter. If we show the results of the temporal correlation coefficient estimations of the speckle for DS1, the above similar behaviors would also be observed. Note that the temporal correlation characteristics of the coherent sea clutter are dominated by the speckle component; thus, its decorrelation time is equal to that of the speckle component.
For the clutter of each range cell, the temporal autocorrelation function of the texture component can be estimated by using the $N_{b}=256$ estimates of $\widehat{\tau}(r, l)$ obtained from (11); that is,

$$
\begin{aligned}
R_{t, \tau} & \left(L_{c} m / 2\right) \\
= & \frac{1}{N_{b}} \sum_{l=0}^{N_{b}-m}\left[\widehat{\tau}(r, l+m)-\widehat{\tau}_{n}\right]\left[\widehat{\tau}(r, l)-\widehat{\tau}_{n}\right],
\end{aligned}
$$

where $\widehat{\tau}_{n}=\left(1 / N_{b}\right) \sum_{l=1}^{N_{b}} \widehat{\tau}(r, l)$. In fact, the temporal correlation estimation of the texture, $R_{t, \tau}(m)$, is obtained only every $L_{c} / 2$ lags because the estimates of $\widehat{\tau}(r, l)$ are obtained by averaging $L_{c}$ pulse samples with a $50 \%$ overlap. Figure 9 shows the estimated results of the temporal correlation coefficient of the texture in the three channels for each range cell for both DS1 and DS2. As discussed previously, the texture estimates have sinusoidal characteristics, and, thus, the temporal correlation of the texture defined in (14) may also present sinusoidal characteristics [34]. In Figure 9, the estimated correlation coefficients exhibit a drop-off at the origin, and then it slowly decays in a periodic manner. For all cases, the initial drop-off trends are similar. For each channel, the periodically decaying trends of the temporal correlations of the texture are broadly similar within a particular range interval (e.g., the interval of 1-200 range cells, the sum channel of DS2), but they are different among different particular range intervals (e.g., between the intervals of 1-200 range cells and of 450-550 range cells, the sum channel for DS2). In addition, for both 


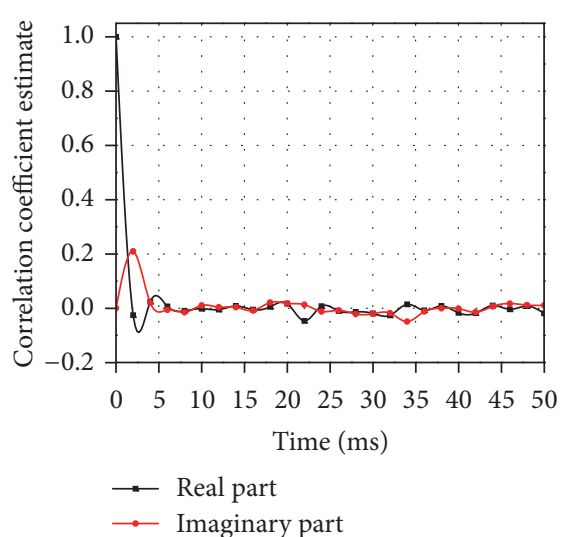

(a)

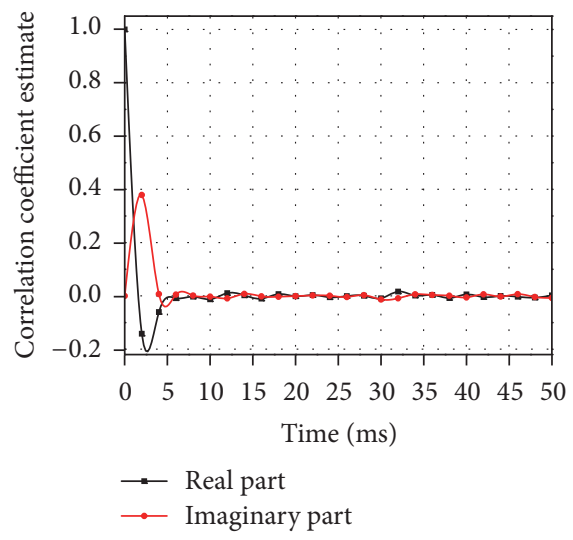

(d)

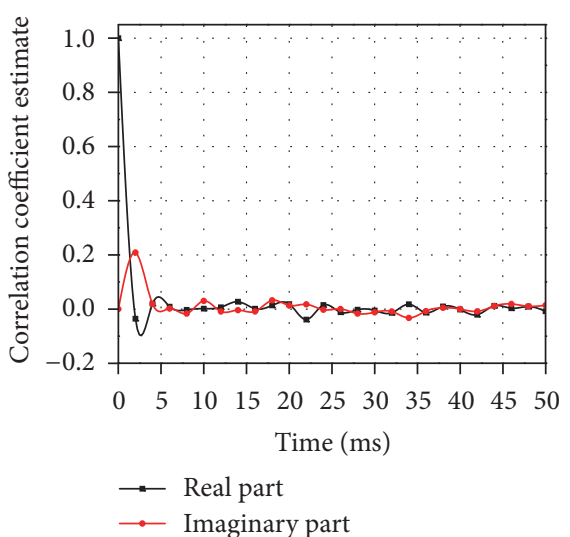

(b)

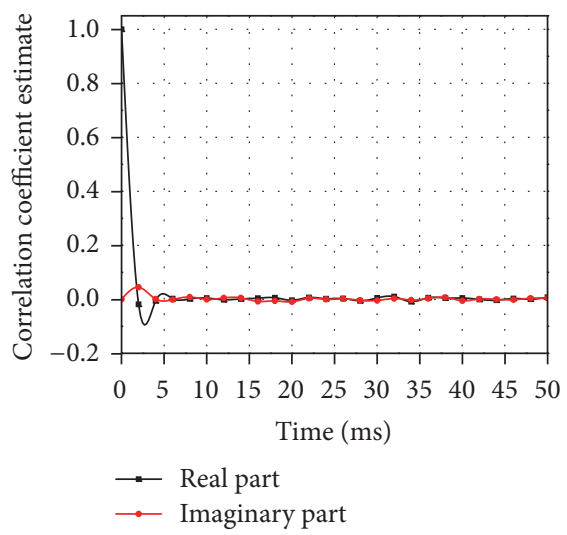

(e)

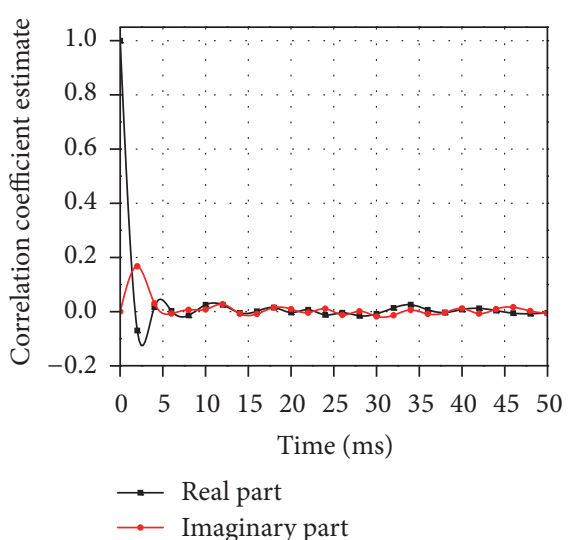

(c)

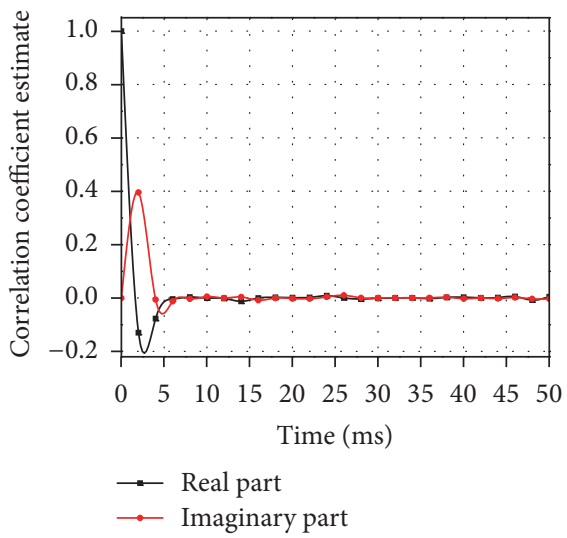

(f)

FIGURE 8: The temporal correlations of the speckle for DS2: ((a)-(c)) sum channel and elevation and azimuth difference channels for the 200th range cell. ((d)-(f)) Sum channel and elevation and azimuth difference channels for the 380th range cell.

DS1 and DS2, these range-time maps show that the temporal correlations of the texture between the elevation difference and sum channels are clearly similar, but there are some differences for those between the azimuth difference and sum channels.

In practice, the correlation operation is often applied as a filter to filter the uncorrelated components and to enhance the periodic components. Because the structures of sea waves and swells appear sinusoidal, the temporal correlations of the texture can be applied to reveal the periodic nature of sea waves and swells. To further examine the dominant periods of sea waves and swells in the three channels, we calculate the absolute value of the Fourier transform (i.e., the power spectra) of the temporal correlations of the texture for each range cell. Figure 10 reports the results of this spectral analysis for DS2. Similar analysis results can also be acquired for DS1. The texture correlation is a real component; thus, its Fourier transform is a real frequency symmetric quantity. It can be observed that, for the three channels, the dominant frequency components can be split into two frequency bands (despite the fact that low frequency band is not comparatively evident), whose frequencies are in the range of $0.035-0.045 \mathrm{~Hz}$ and $0.15-0.35 \mathrm{~Hz}$, respectively. It is believed that the swell contribution is related to the band of low frequencies and the sea wave contribution is related to the high frequencies. Hence, the corresponding periods are approximately 22.2-28.6 s for the swell and are approximately 2.9-6.7 s for the sea waves. It is evident that, for each channel, the dominant frequencies of sea waves are nearly similar within a particular range interval (e.g., the interval of 1-200 range cells, the sum channel), but they are different among different particular range intervals (e.g., between the intervals of 1-200 range cells and of 450-550 range cells, the sum channel). This phenomenon coincides with the variational characteristics of the temporal correlations of the texture shown in Figure 9. Moreover, the dominant periodic characteristics of the temporal correlations of the texture component are quite similar for the elevation difference and sum channels, but they are partly different between the azimuth difference and sum channels.

To investigate the overall behavior of each channel, the temporal correlations of the texture and their power spectra are averaged over all range cells. The results are shown in Figures 11 and 12 for DS1 and DS2. It is evident that the averaged power spectra also show two peaks. Although there are some faint differences, both the averages of the 


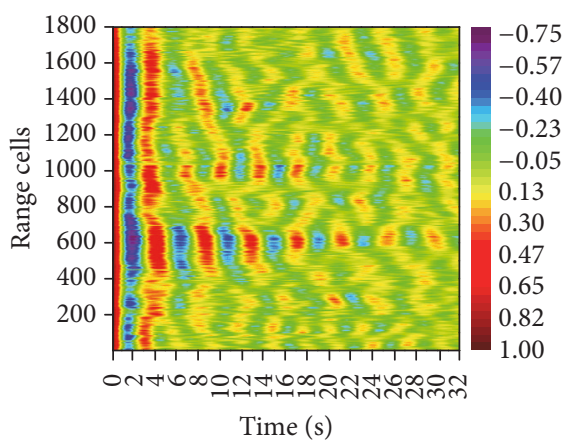

(a)

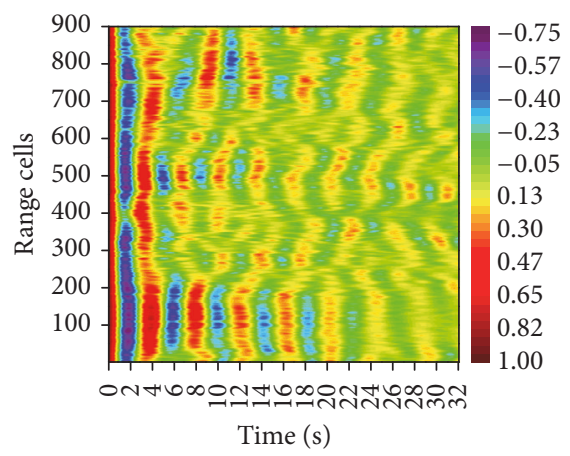

(d)

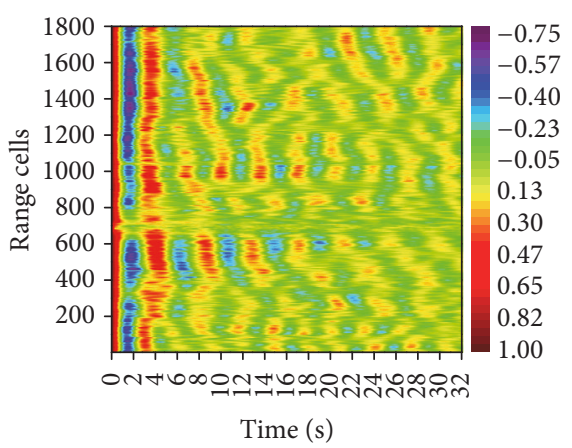

(b)

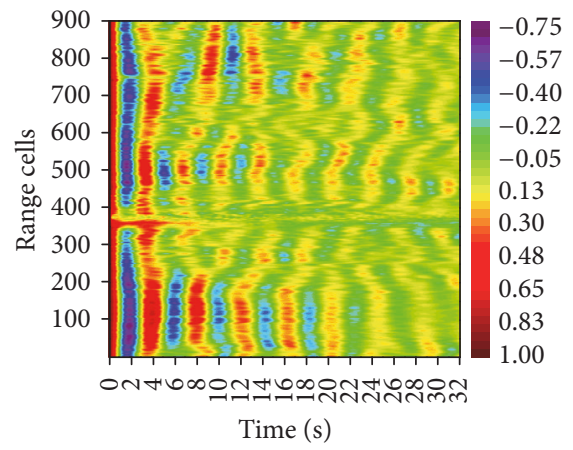

(e)

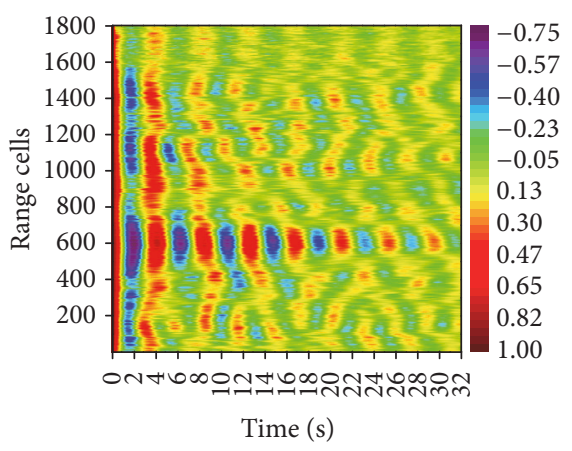

(c)

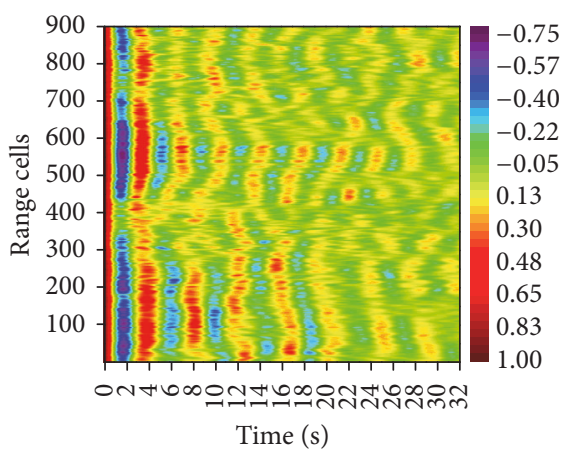

(f)

FIGURE 9: The temporal correlations of the texture: ((a)-(c)) sum channel and elevation and azimuth difference channels for DS1. ((d)-(f)) Sum channel and elevation and azimuth difference channels for DS2.

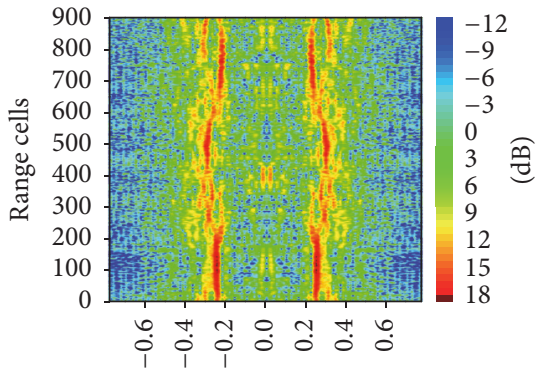

Frequency $(\mathrm{Hz})$

(a)

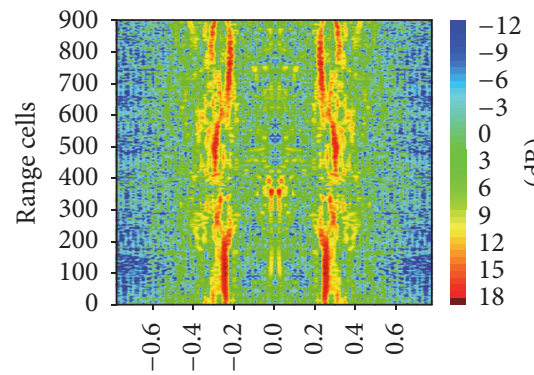

Frequency $(\mathrm{Hz})$

(b)

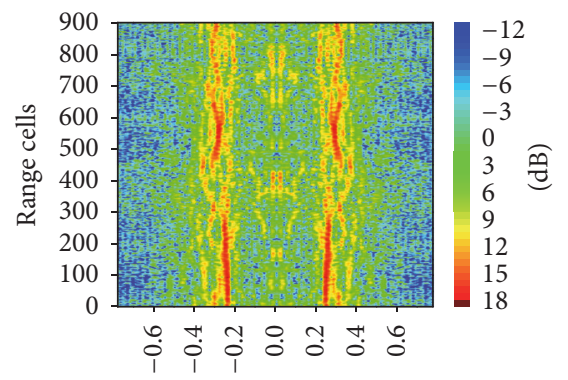

Frequency $(\mathrm{Hz})$

(c)

FIGURE 10: Power spectra of the temporal correlations of the texture for DS2: (a) sum channel. (b) Elevation difference channel. (c) Azimuth difference channel.

temporal correlations of the texture and of their power spectra maintain overall consistency for all channels of each dataset. However, for these two datasets, their illumined sea patches were quite close, their recording times were nearly consecutive, and the differences of their grazing angles are not great enough to cause the significant differences between the temporal correlations of their texture. Therefore, in Figures 11 and 12, for DS1 and DS2, the plots of the averages of the temporal correlations of the texture appear similar, and the closely dominated frequencies are presented in the plots of the averaged power spectra. The dominated frequencies can be approximately $0.03 \mathrm{~Hz}, 0.25 \mathrm{~Hz}$, and $0.3 \mathrm{~Hz}$; thus the corresponding periods of the dominated swell and waves are approximately $33.3 \mathrm{~s}, 4 \mathrm{~s}$, and $3.33 \mathrm{~s}$.

4.3. Estimation of the Spatial Correlation Functions of Sea Clutter. As described in previous subsections, the clutter exhibits a periodic varying nature with range and time. We can extend the calculating methods of the temporal correlations to estimate the spatial correlations for the speckle and texture components. There are no apparent differences in the estimations between DS1 and DS2; thus, we report here 


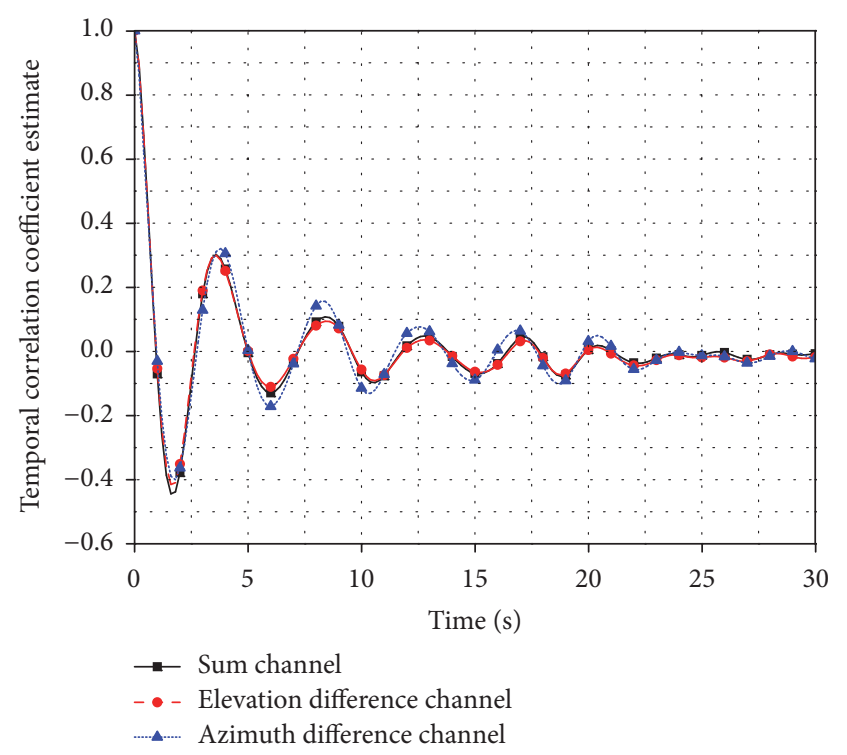

(a)

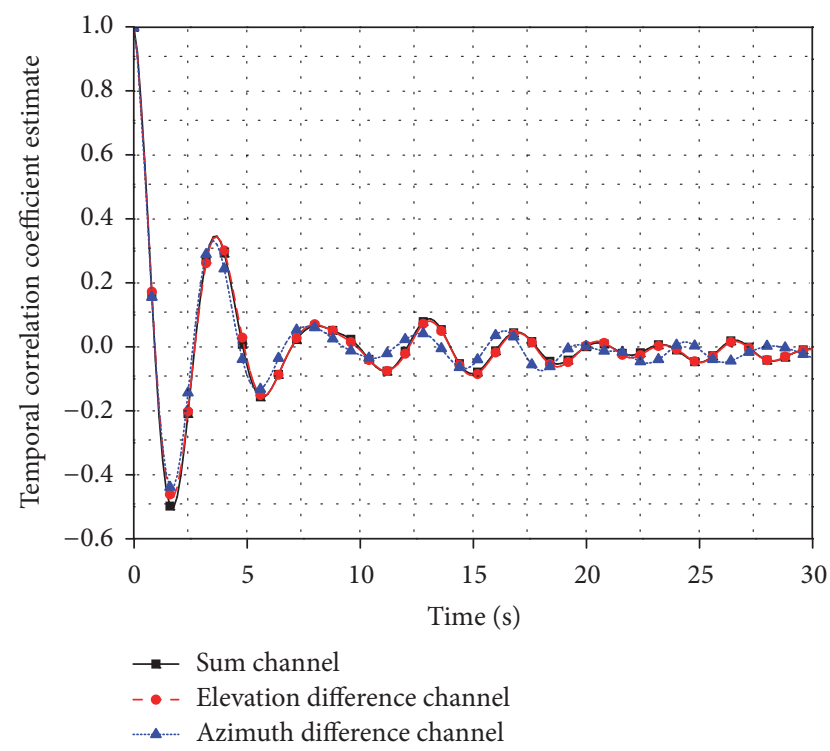

(b)

FIGURE 11: The averages of the temporal correlations of the texture for DS1 and DS2: (a) DS1. (b) DS2.

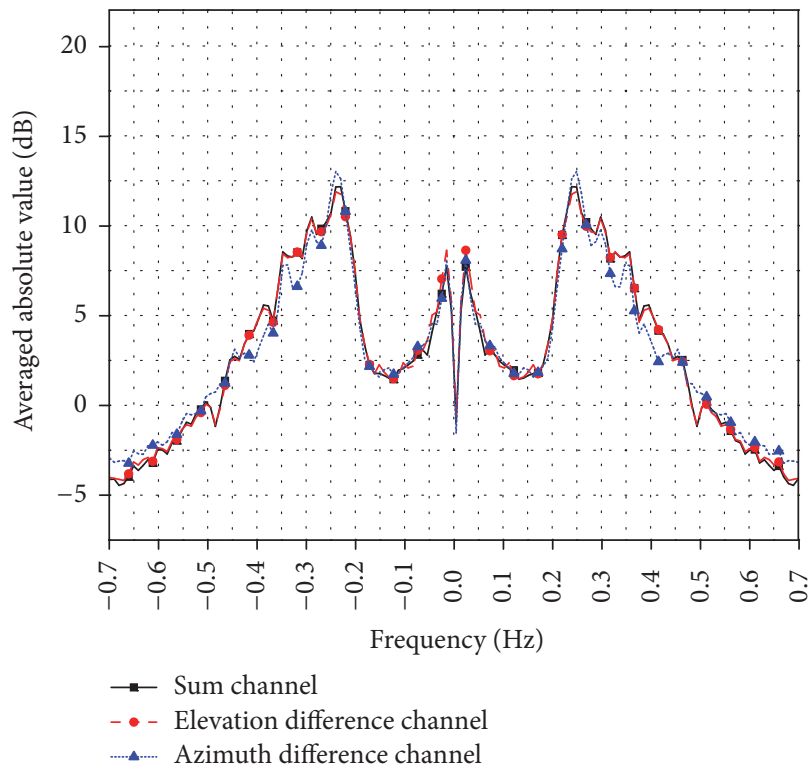

(a)

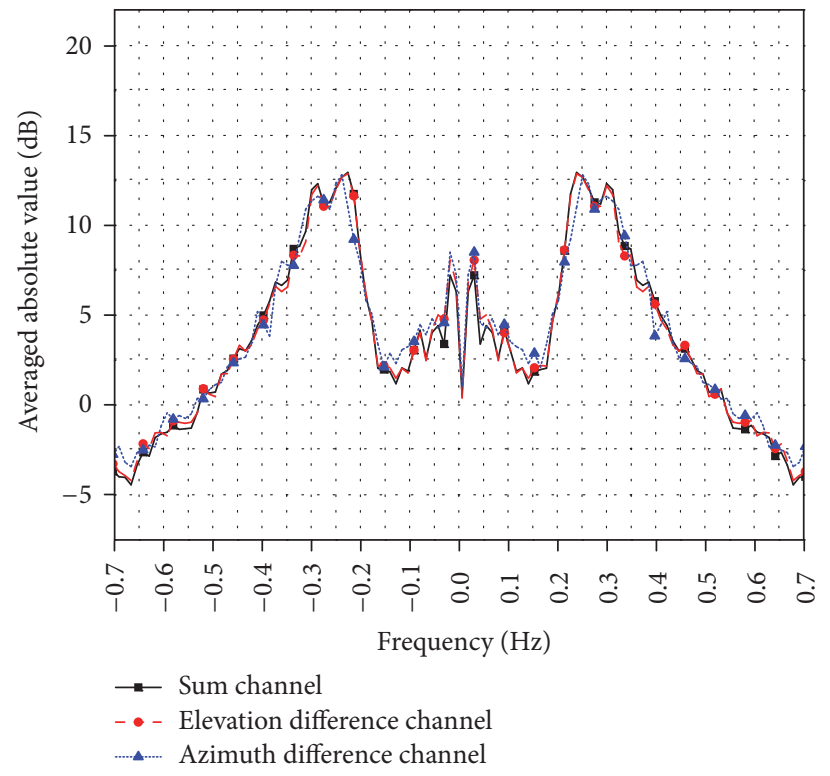

(b)

FIGURE 12: The averages of the power spectra of the temporal correlations of the texture for DS1 and DS2: (a) DS1. (b) DS2.

only the results for DS2. In Figure 13, we present the results for the spatial correlation of the speckle. For each pulse, the entire range sequence of the estimated speckle is divided into 7 bursts of 256 samples each, with an overlap between adjacent bursts of $50 \%$. The final results are obtained by averaging the estimation results over 1024 successive pulses. It is evident that, for the high-resolution radar, the speckle component is spatially correlated in a short range. The imaginary parts of the spatial correlation coefficients of the speckle are low, and thus, they can be neglected. This indicates that the in-phase and quadrature components of the clutter are uncorrelated in range [35]. Additionally, note that, for three-channel clutter, the real parts of the estimates are very similar.

It can be easily realized from the previous discussions that, for different range cells, the intensities of the texture estimates are modulated nonuniformly by the gains of the transmitter and receiver antennas. This may strongly affect the analysis results of the spatial correlation of the texture. 


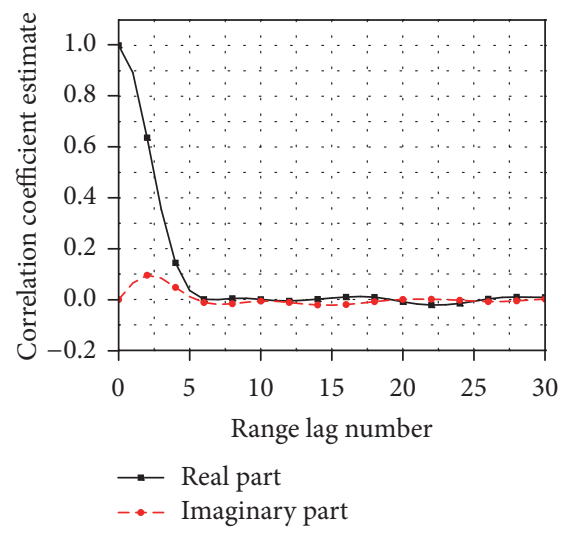

(a)

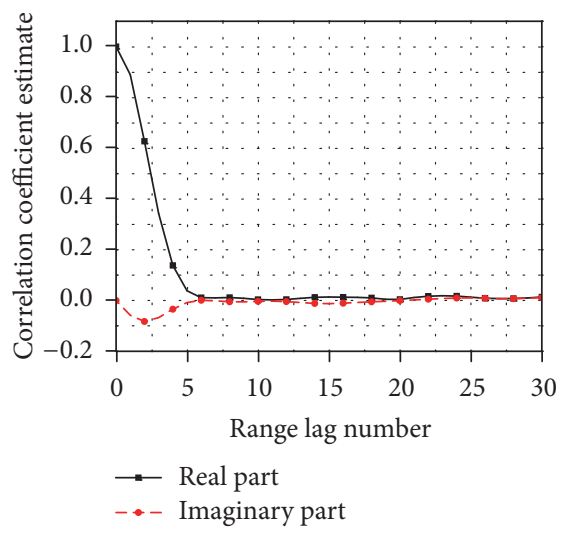

(b)

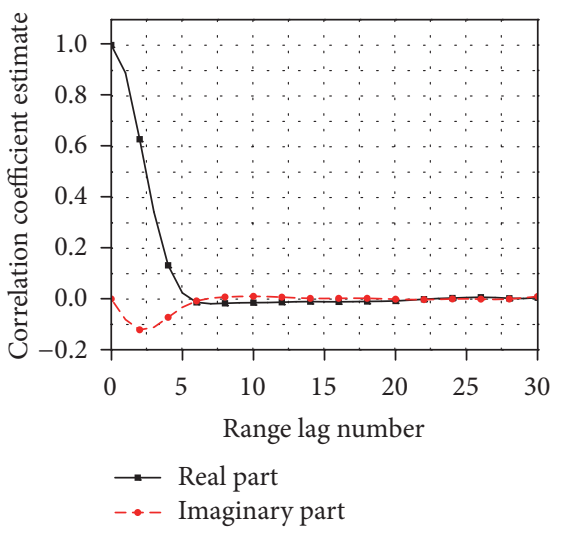

(c)

FIGURE 13: The spatial correlations of the speckle for DS2: (a) Sum channel. (b) Elevation difference channel. (c) Azimuth difference channel.

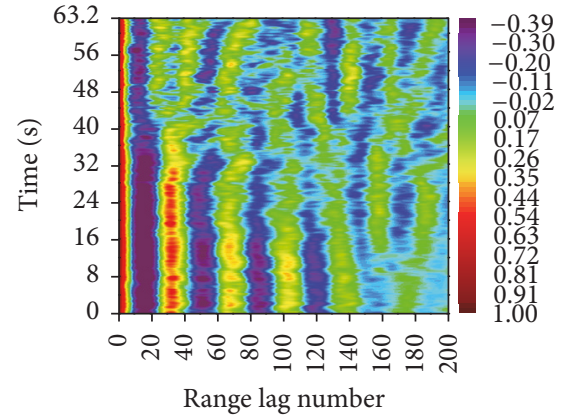

(a)

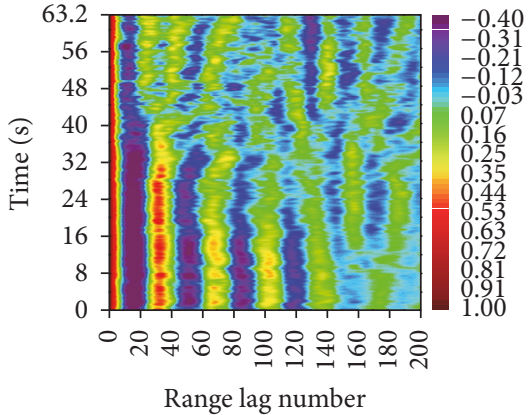

(b)

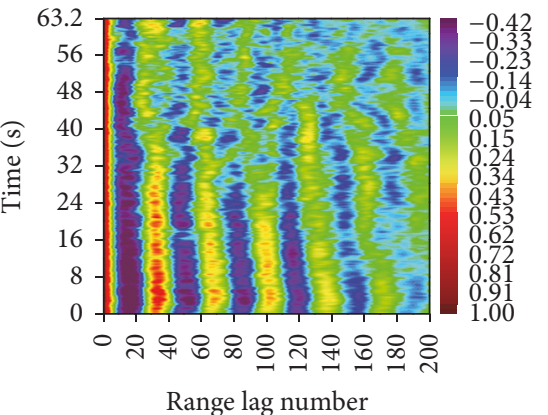

(c)

Figure 14: The spatial correlations of the texture for DS2: (a) Sum channel. (b) Elevation difference channel. (c) Azimuth difference channel.

Therefore, to reduce this effect, for each range cell, the texture estimates of the three channels are, respectively, normalized by the clutter powers of the respective channels in the corresponding range cell. The spatial correlation function for the texture components at different range lags is then calculated via the formula given in (14). Figure 14 shows the results of this spatial correlation analysis. These estimations are obtained from range cells 51 to 307 of DS2 (equivalent to a range interval of $192 \mathrm{~m}$ ). As shown in the figures, for all estimated correlation coefficients, there is initially a similar drop-off, which is followed by a slowly decaying process. The periodic behavior of the spatial correlation of the texture in the decaying process is clearly visible for the three channels for both datasets. However, note that, for the spatial correlation of the texture of each channel, the form of the periodic behavior is not constant but has a changing period. Clearly, for the elevation difference and sum channels, their spatial correlations of the texture components are very similar, but compared to them, there is some difference for that of the azimuth difference channel.

To further investigate the periodic behavior, a similar analysis approach for the temporal correlation is used to obtain the power spectra of the spatial correlations of the texture. Figure 15 shows the analysis results of the data in Figure 14. Here, the dominant frequencies refer to the periodic lag numbers of the spatial correlation. They are closely associated with the wavelengths of the sea waves and swells. It is very evident that, between approximately 32 and $45 \mathrm{~s}$ into the time history, a very large variation in the dominant periodic components is apparent: the preexisting dominant components with close frequencies and large wave energy disappear, and other dominant components with dispersive frequencies and relatively small wave energies subsequently appear. This variation is thought to be a result of the occurrence of wave breaking events. The occurrence of the wave breaking events can redistribute the wave energy further; thus, the structure of the sea surface experiences huge variations, which are reflected in the temporal variations of the spatial correlation of the texture shown in Figure 14. However, for the elevation difference and sum channels, the dominant periodic components of the texture are quite similar. Although there are some different periodic components between the azimuth difference and sum channels, the overall variation trends of the structures of sea surface area illuminated by them are similar. For the three channels, the frequencies of the dominant components are in the range of 


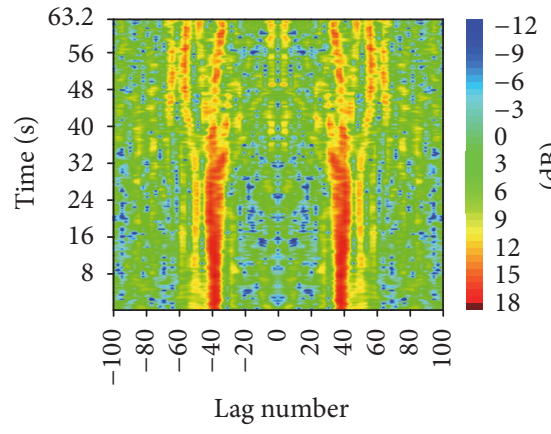

(a)

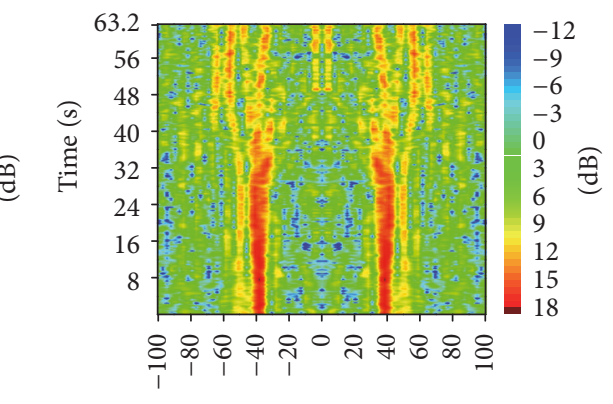

Lag number

(b)

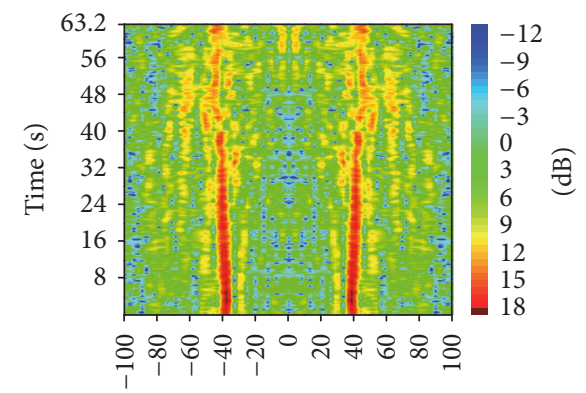

Lag number

(c)

Figure 15: Power spectra of the spatial correlations of the texture: (a) Sum channel. (b) Elevation difference channel. (c) Azimuth difference channel.

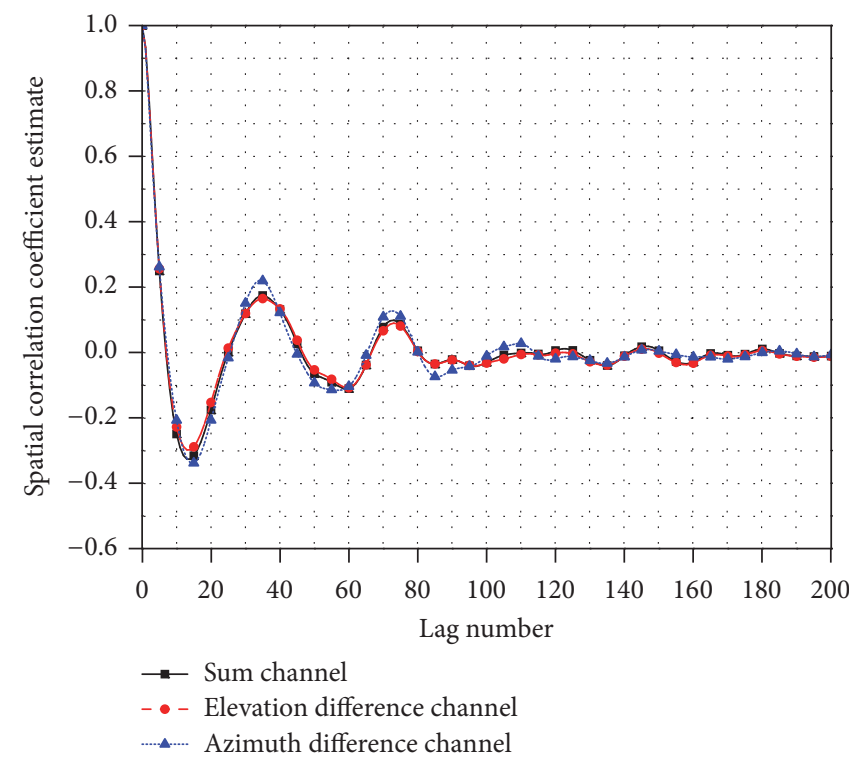

(a)

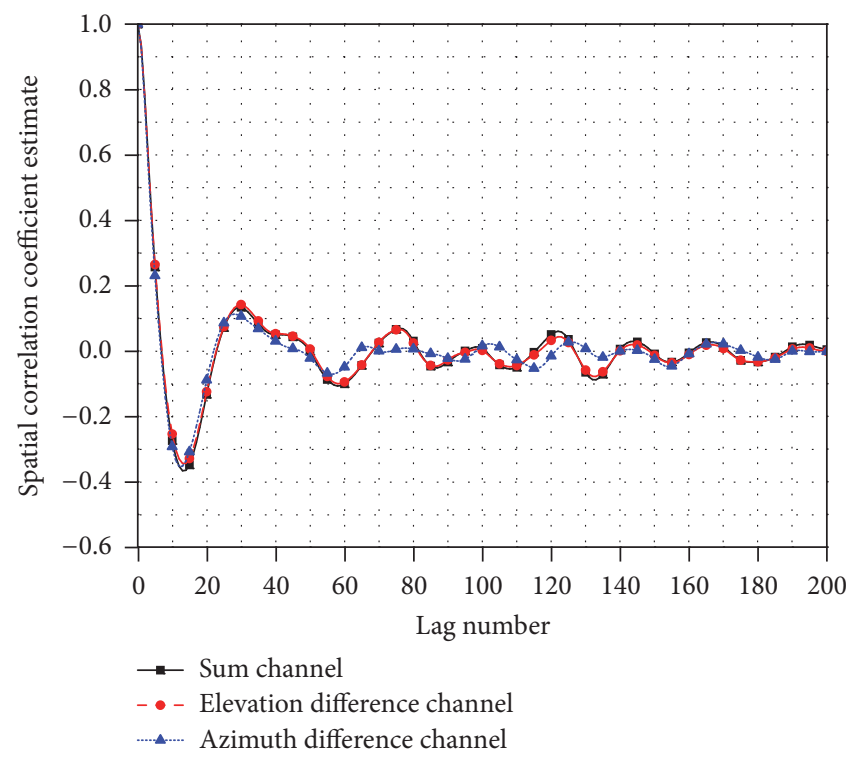

(b)

FIGURE 16: The averages of the spatial correlations of the texture for DS1 and DS2: (a) DS1. (b) DS2.

30-70. According to the viewing geometry (i.e., the grazing angle and the aspect angle with respect to the wind direction), we can obtain that the estimated wavelengths of the dominant waves are approximately in the range of $17.5-40.8 \mathrm{~m}$.

Similarly, Figures 16 and 17 report the averages of the spatial correlations of the texture and of their power spectra for DS1 and DS2 to investigate the overall behavior of each channel. It can be observed that although there are some differences for the individual spatial correlations of the texture among the three channels, the averages of the spatial correlation coefficients of the texture and of their power spectra maintain overall consistency for all channels of each dataset. As demonstrated in [34], the wavelengths of the sea waves are closely related to their periods. Consequently, for the spatial correlation of the texture, Figures 16 and 17 display similar behaviors with the temporal correlations of the texture; that is, the averages of the spatial correlation coefficients of the texture and of their power spectra are very similar for these two datasets. We can see that the dominant frequencies for DS1 are approximately 35.6 and 53.5 and those for DS2 are approximately 33.1 and 53.5. Accordingly, the corresponding wavelengths of the dominant waves for DS1 are approximately $20.5 \mathrm{~m}$ and $31 \mathrm{~m}$, and those for DS2 are approximately $19.3 \mathrm{~m}$ and $31.2 \mathrm{~m}$. It is very evident that the wavelengths of the dominant waves estimated from these two datasets are coincident.

Although the overall behaviors of both the temporal and spatial correlations are similar for DS1 and DS2, some subtle differences can still be observed in their plots after a meticulous comparison. However, it is very difficult to determine that these differences are caused by the dynamic nature of the sea surface, by the different measurement parameters of grazing angle, or by both of them. The effects of the grazing angle on the correlation characteristics of sea 


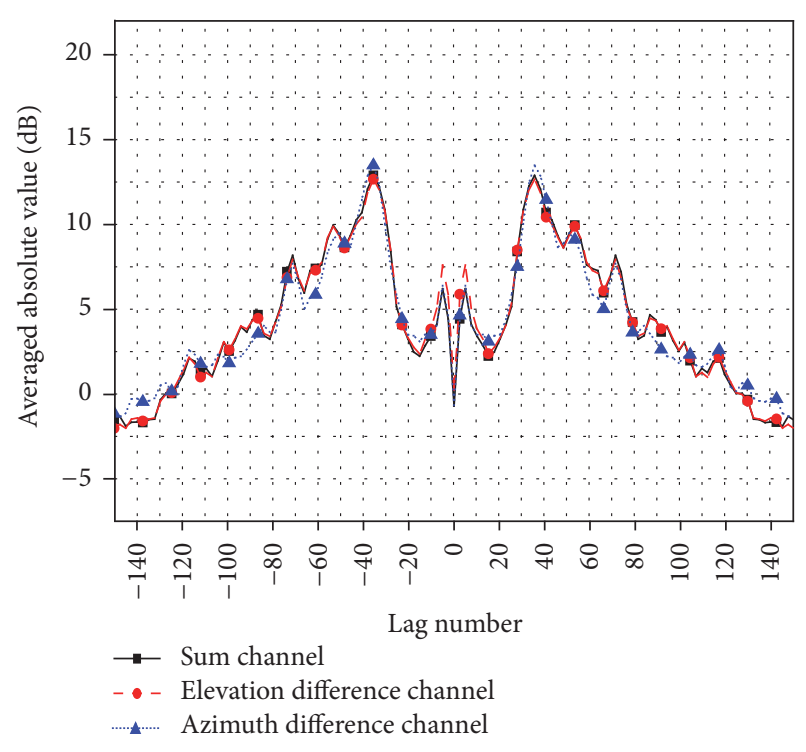

(a)

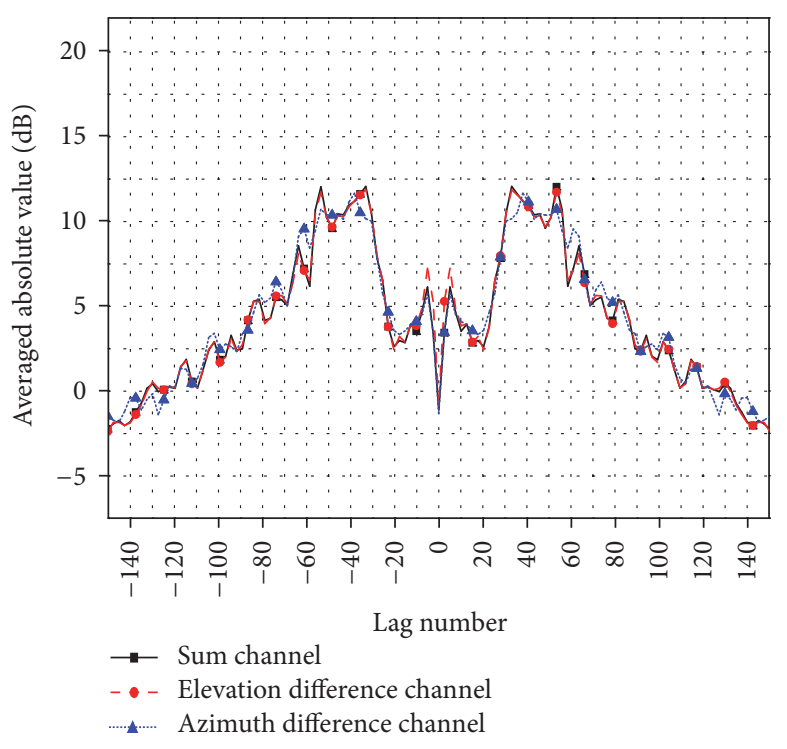

(b)

FIGURE 17: The averages of the power spectra of the spatial correlations of the texture for DS1 and DS2: (a) DS1. (b) DS2.

clutter can therefore not be deduced from these above results. As stated by Dong in [34], the sea surface may be modeled as a combination of correlated scatterers (periodic undulating surfaces) and random and uncorrelated scatterers (whitecaps, breaking waves, and so forth). In practice, the correlation characteristics of sea clutter are dominated by the correlated scatterers. It is well known that the dominant radar backscatter varies with the variation of the grazing angle, which may cause the variation of the correlation characteristics of sea clutter. For the same sea patch, however, the power spectra of the correlations may occasionally exhibit similarly dominated frequencies even if in the different grazing angles.

4.4. Estimation of the Cross-Correlation Functions of Sea Clutter between the Sum and Difference Channels. It is necessary to investigate the clutter cross-correlation characteristics between the sum and difference channels by a method similar to that above. For each range cell, the cross-correlation coefficient is calculated via the following formula:

$$
\rho_{c}=\frac{\sum_{n=1}^{N}\left(x_{d}(n)-\bar{x}_{d}\right)\left(x_{s}(n)-\bar{x}_{s}\right)^{*}}{\sqrt{\sum_{n=1}^{N}\left(x_{d}(n)-\bar{x}_{d}\right)^{2}} \sqrt{\sum_{n=1}^{N}\left(x_{s}(n)-\bar{x}_{s}\right)^{2}}}
$$

where $x_{d}(n)$ and $x_{s}(n)$ denote the texture or speckle components in the difference and sum channels, respectively, and $\bar{x}_{d}$ and $\bar{x}_{s}$ are the mean values of $x_{d}(n)$ and $x_{s}(n)$, respectively.

Figures 18 and 19 report the cross-correlation results for the texture and speckle, respectively. It is very evident from visual inspection of these figures that, between the elevation difference and sum channels, the cross-correlation coefficients of both the texture and speckle are close to 0.95 in the range cells where the CNRs of two channel are higher than $5 \mathrm{~dB}$. The clutter returns in these two channels can be considered to be completely correlated. Between the azimuth difference and sum channels, however, the crosscorrelation coefficients of the speckle are very low, and the texture components are also only partly correlated. Therefore, the clutter returns in these two channels are partly correlated or uncorrelated. The results in Figure 18 go a long way in explaining the presences of the similarities and differences among the preceding texture correlations of the three channels.

The texture cross-correlations between the difference and sum channels are affected by both the proportions of the common effective illumination area of the receiver antennas and the channel CNRs. In the high-resolution monopulse radar system, the corresponding elevation off-boresight angle of each range cell is bounded due to the range sampling. Thus, the proportions and the channel CNRs vary with range. In elevation direction, as the range cells gradually approach the range region corresponding to the boresight axis, the effective illumination area of individual range cell of sum channel increases but that of difference channel decreases. As a consequence, the proportion of their common effective illumination areas reduces sharply. Moreover, the elevation difference channel has low CNR in the middle range region corresponding to the boresight axis. Consequently, in Figure 18, there is a sharp drop in the correlation coefficients for elevation direction.

\section{Spectral Analysis}

In the coherent radar system, Doppler processing is widely applied not only as a coherent accumulation technique to improve the SCNR (signal to clutter plus noise ratio) but also as a coherent filter to obtain the Doppler frequency information. In particular, if the target's radial velocity is sufficiently high and if the clutter Doppler spectrum is centered at low 


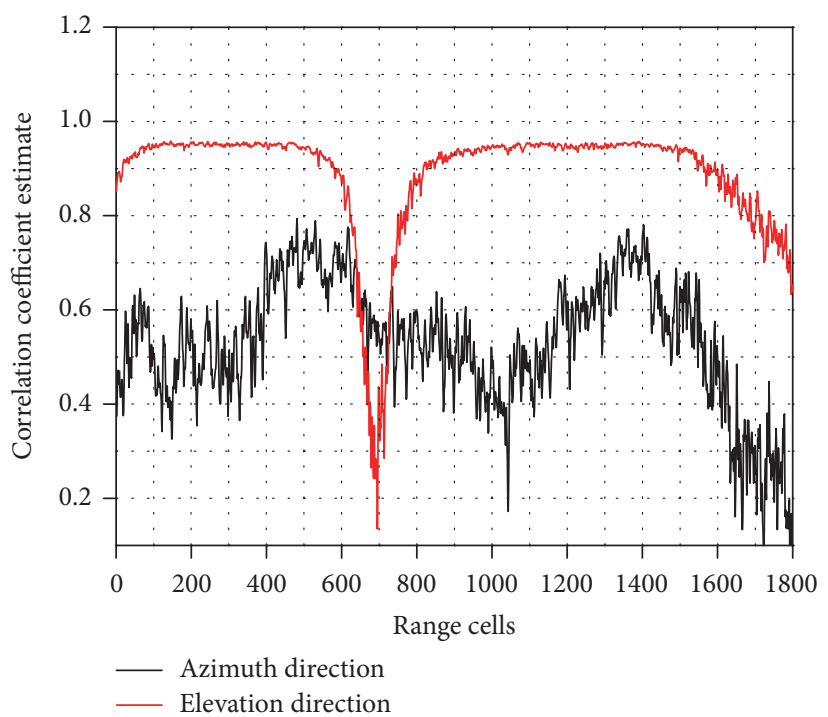

(a)

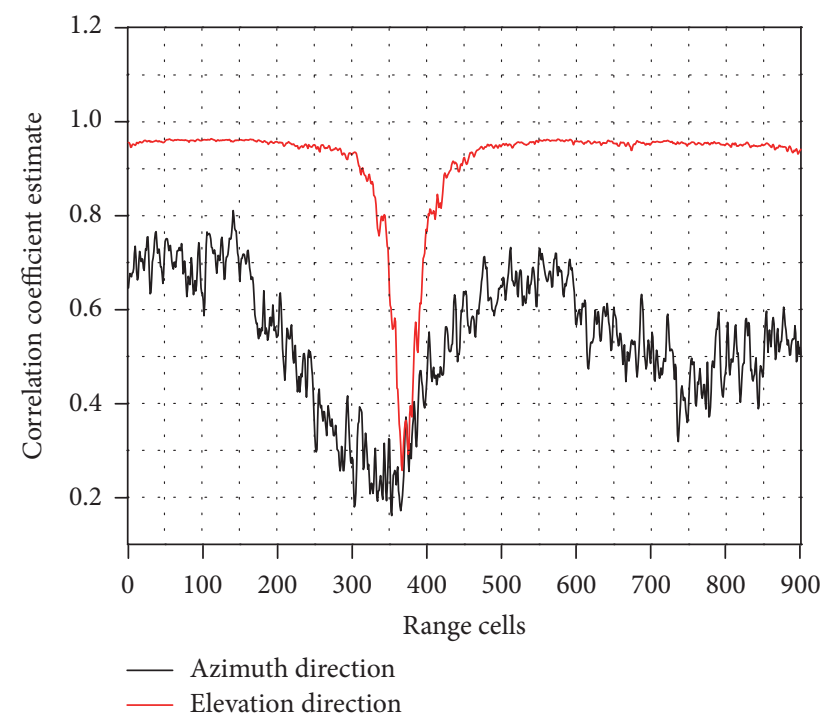

(b)

FIGURE 18: The texture cross-correlations between the difference and the sum channels for DS1 and DS2: (a) DS1. (b) DS2.

frequencies, the targets can be distinguished from clutter in the Doppler domain. However, some targets of interest will have Doppler shifts that are not significantly different from the Doppler spectrum of the clutter. The Doppler spectrum of the target and that of clutter are now mixed together. In these cases, having a comprehensive understanding for the characteristic of the clutter Doppler spectrum is very helpful in designing specific signal processing algorithms for clutter suppression and for target detection [53-56]. Moreover, the proper model of the clutter Doppler spectrum is essential for the accurate simulation of the coherent clutter returns $[32,33,37]$. In this section, the Doppler spectrum properties of the recorded sea clutter are discussed.

For each range cell, the Doppler spectra are estimated by computing the 512-point fast Fourier transform (FFT) over a sliding window of 256 pulse samples with an overlap of $50 \%$. A $-55 \mathrm{~dB}$ Dolph-Chebyshev weighting function is used in the time domain. The 512-point FFT provides the Doppler spectrum in the frequency interval $-250-250 \mathrm{~Hz}$. In this frequency interval, the Doppler spectra suffer from aliasing, that is, the edges of the spectra as the aliased components wrap into the sampled frequencies. Hence, before the Doppler spectra are analyzed, the unaliased Doppler spectra need to first be obtained by shifting the aliased frequency components to the correct positions in the spectra. It is well known that the Doppler spectrum of clutter may exhibit timevarying and range-varying behaviors. A contemporary study of the Doppler spectrum evolution along range and time is therefore conducted. The data in this section are primarily illustrated using DS2, and similar results can be obtained from DS1.

The clutter spectra contain three-dimensional information, namely, the range, time, and frequency information. Generally, the two-dimensional range Doppler and time
Doppler maps are applied to illustrate the spectra characteristics. They can be, respectively, rebuilt from threedimensional spectra by squeezing the spectra within one same time interval and within one same range cell. Figures 20 and 21 show examples of them for the three channels. It can clearly be observed that the Doppler centroids (mean Doppler frequencies) of individual spectra are nonzero due to the internal motion of the sea. As described in [37, 38, 50, 57], for the three channels of both, the range- or time-varying natures of the spectra are very evident. Furthermore, these varying natures are mainly reflected in two aspects: the clutter intensity varies periodically with range in the integrated range Doppler spectra or with time in the integrated time Doppler spectra, and the form of the individual spectrum varies in range or time with a changing Doppler centroid and a fluctuating spectrum width (the standard deviation of the individual spectrum). As observed empirically in [37, 38, 50], the varying behavior of the individual spectrum shape appears to be associated with the underlying modulation of clutter mean intensity. Thus, the entire spectra are further analyzed to investigate the variations in spectrum shape, Doppler centroid, and spectrum width and to discuss the dependencies of them on the average spectrum intensity in more detail.

In the foregoing analysis, it is empirically shown that the local clutter power can be approximately constant within several neighboring range cells along the range dimension and during the short time intervals along the time dimension. The spectra of sea clutter within the neighboring range cells and during the short time intervals can also be considered to exhibit similar behavior. To obtain a more stable observation of the underlying tendency for clutter data, the spectra are averaged twice; that is, the first estimated spectra are achieved by averaging over the groups of six successive spectra with an overlap of 50\% along time, and then they are averaged over 


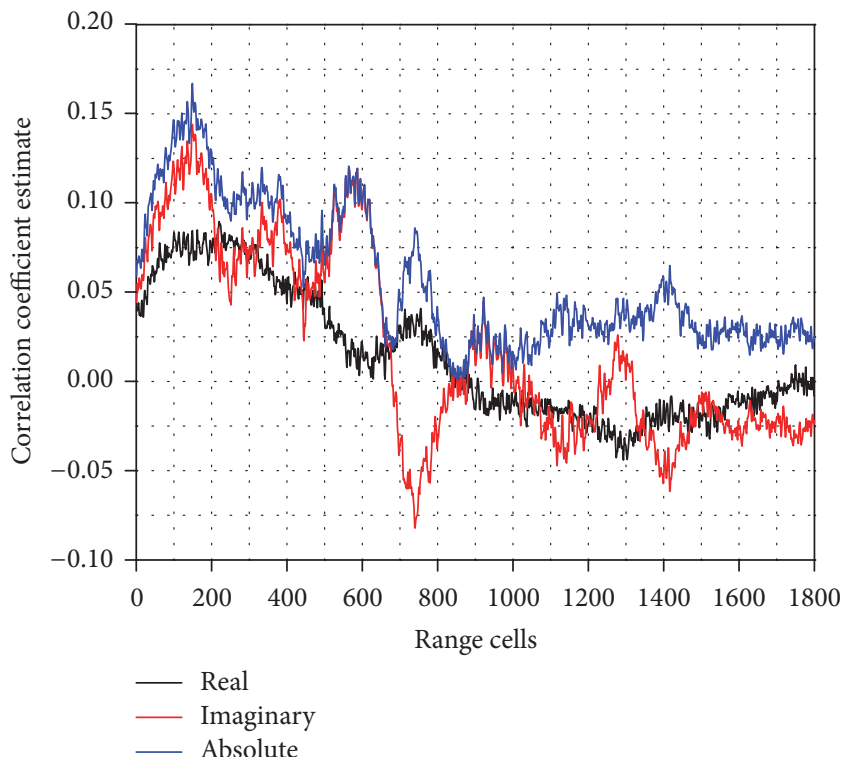

(a)

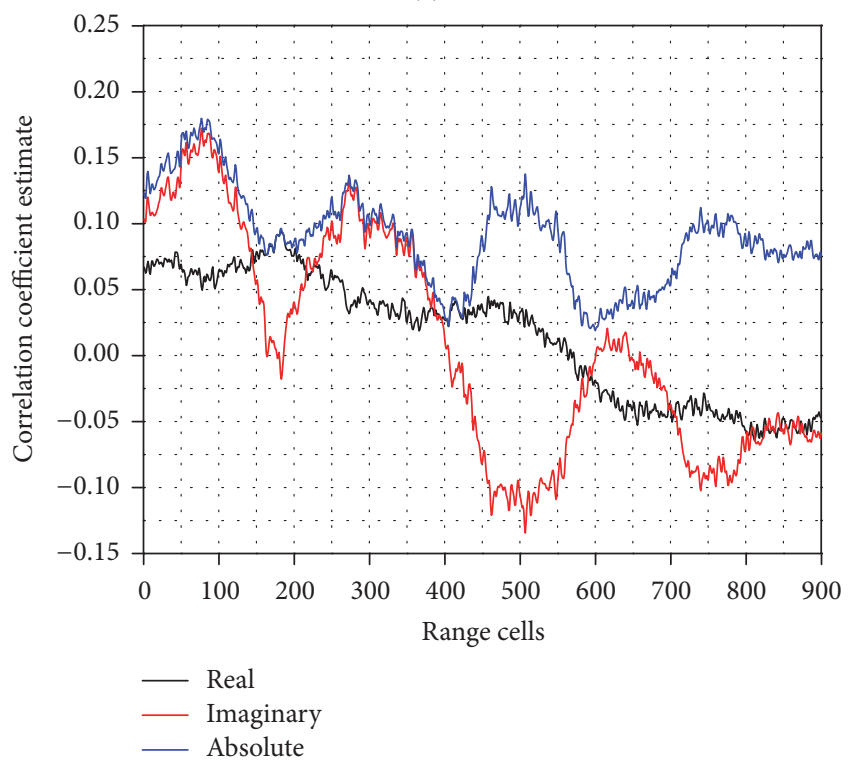

(c)

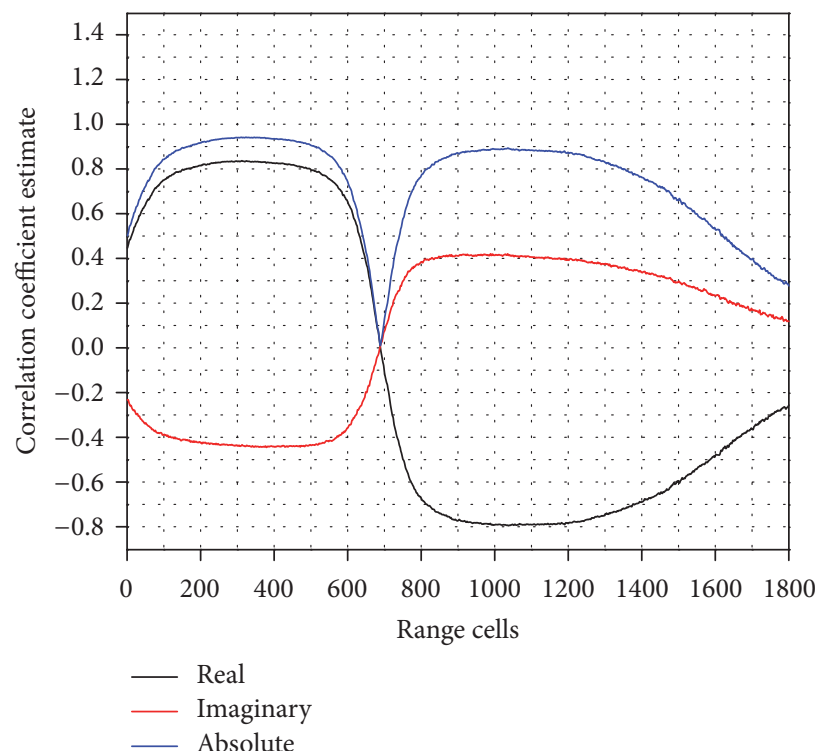

(b)

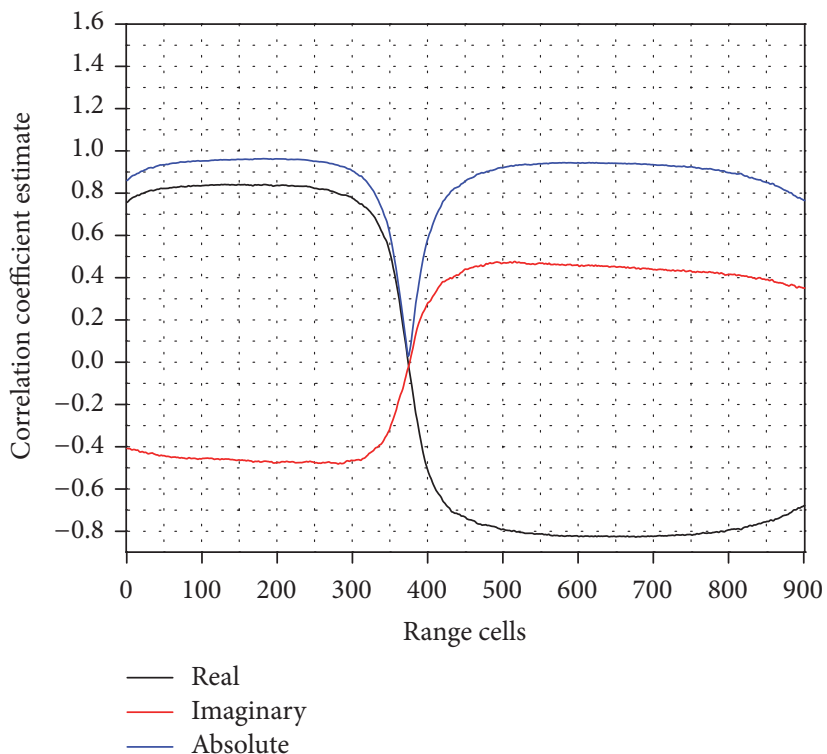

(d)

FIGURE 19: The speckle cross-correlations between the difference and the sum channel for DS1 and DS2: (a) Azimuth direction for DS1. (b) Elevation direction for DS1. (c) Azimuth direction for DS2. (d) Elevation direction for DS2.

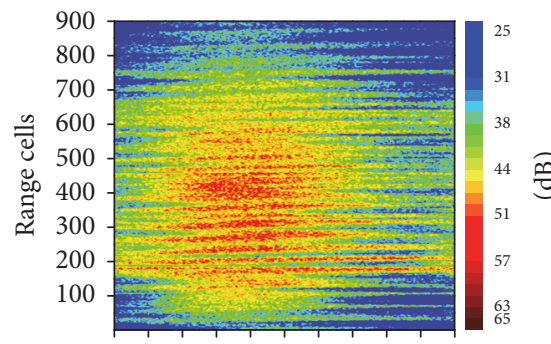

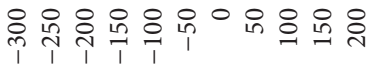

Doppler frequency $(\mathrm{Hz})$

(a)

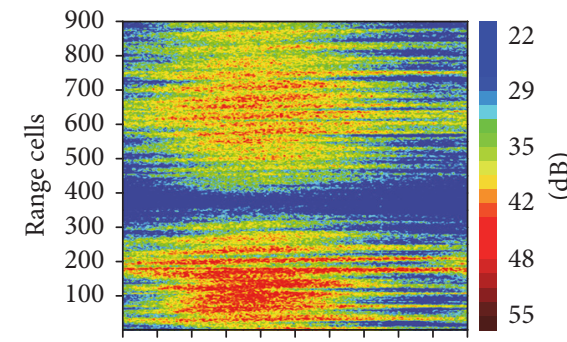

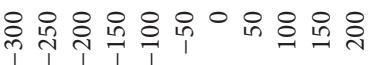

Doppler frequency $(\mathrm{Hz})$

(b)

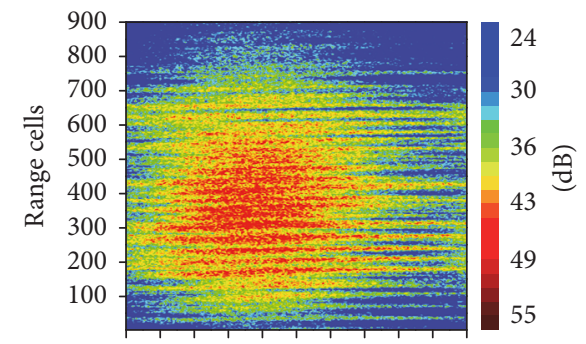

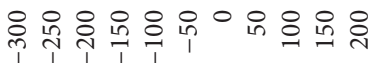

Doppler frequency $(\mathrm{Hz})$

(c)

Figure 20: Examples of range Doppler maps, the first time burst: (a) sum channel. (b) Elevation difference channel. (c) Azimuth difference channel. 


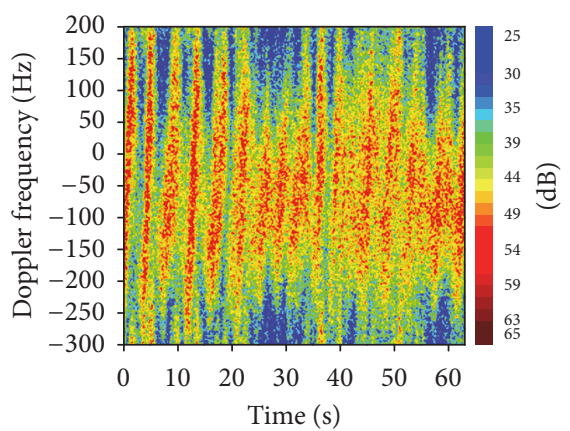

(a)

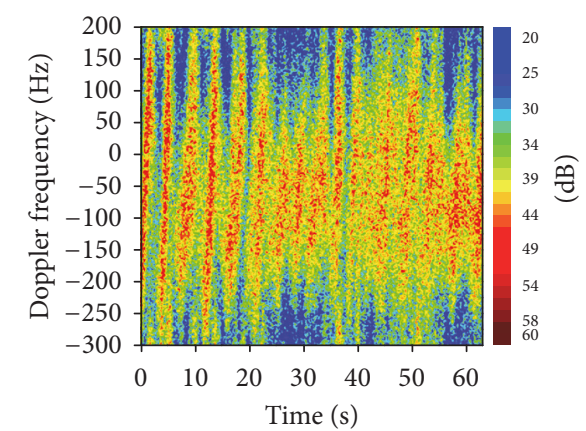

(b)

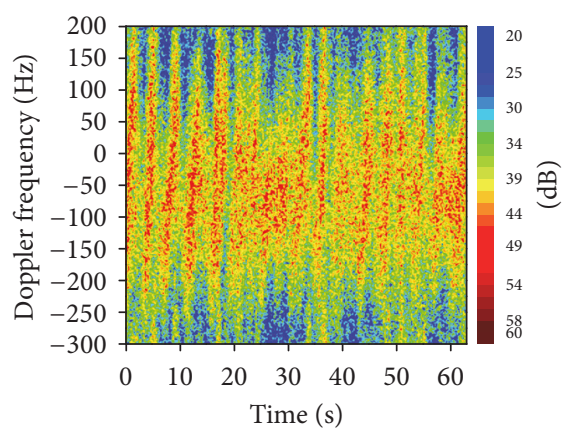

(c)

FIGURE 21: Examples of time Doppler maps, the 202nd range cell: (a) sum channel. (b) Elevation difference channel. (c) Azimuth difference channel.

the groups of three successive spectra with an overlap of one spectrum along range. This would smooth local fluctuations of the spectrum without affecting the measurement of the longer-term trends. Consequently, for each range cell, the entire time sequence of the spectra has been divided into 81 time bursts.

The shape of an individual spectrum is an essential parameter required to model the clutter spectrum. In the open literature, it has been experimentally confirmed that a spectrum model with a Gaussian shape can provide a good fit to the estimated clutter spectra. Similarly, this Gaussianshaped spectrum model is used here to fit the estimated normalized PSDs. The normalized PSDs are achieved by normalizing the spectrum intensity to a peak value of unity. The form of this Gaussian-shaped PSD can be expressed as $[33,37,38,58]$

$$
G\left(f, m_{f}, s\right)=\frac{x}{\sqrt{2 \pi} s} \exp \left[-\frac{\left(f-m_{f}\right)^{2}}{2 s^{2}}\right]
$$

where $x$ is the underlying intensity of the sea clutter, $m_{f}$ is the mean Doppler frequency, and $s$ is the spectrum width.

Figure 22 shows some representative examples of individual spectra fitted by this Gaussian-shaped PSD for three channels. The unknown parameters have been estimated by employing a nonlinear least squares method to solve the optimization of the RMSE of the fit to the theoretical spectral model. It can be observed that the Gaussian-shaped spectrum can provide a good qualitative fit for all individual spectra and for all channels. Similar results can be obtained for other spectra and for another dataset. As expected, the spectra forms of the same channel in different range cells and/or in different time bursts appear to be different. In the foregoing correlation analysis, we know that the texture components are intermediately correlated between the azimuth difference and sum channels and that they are highly correlated between the elevation difference and sum channels. Therefore, in these representative examples, for the same range cell and the same time burst, the spectra shapes are apparently different between the azimuth difference and sum channels, but they are very similar between the elevation difference and sum channels. These features highlight again that the spectra forms have a strong dependence on the underlying intensity. Furthermore, a common Gaussian-shaped fit can be used to approximately model the spectra in the sum and elevation difference channels. To further investigate the range- and time-varying natures of this Gaussian-shaped spectrum model, its unknown parameters (i.e., the mean Doppler and the width of individual spectrum) are estimated for all individual spectra over entire range and time intervals.

As already suggested in $[37,38,50]$, there is a strong correlation between the Doppler centroid and the average spectrum intensity. Moreover, the width of the Doppler spectra also appears to display a nonelected correlation with the average spectrum intensity. To further quantify the dependency relationships, the centroid and width of the Doppler spectra are considered as functions of the average spectrum intensity. A linear relationship between the average intensity and the Doppler centroid has been proposed to describe either the time-varying or range-varying natures of the spectra in $[37,38]$. It is of considerable interest to further extend this model to represent the time-varying and range-varying natures of the Doppler spectra simultaneously. The parameter estimates of Doppler spectra evolutions along range are first analyzed for each time burst in a similar manner to [37]. Figure 23 shows the results of this analysis for the spectra taken from the 1st, 20th, and 50th time bursts over entire range cells for the sum channel. Figure 24 shows the equivalent plots for the azimuth difference channel. The least-mean-square error straight-line fits to these parameter estimates are also shown in the figures.

It can be qualitatively observed from Figures 23 and 24 that both the centroid and the width of the Doppler spectra are correlated with the average spectrum intensity in both channels. As suggested in [37, 38], although there is the presence of a quite large fit error in the fit results, the approximately linear relationships between the average intensity and these spectra parameters may still be valid to reproduce many of the features of the Doppler spectra, and this will be shown in the following. The original model proposed in [37] can therefore be extended to represent the 


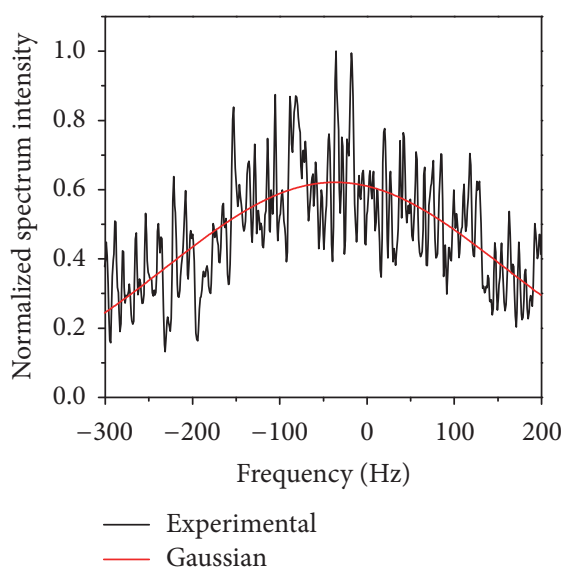

(a)

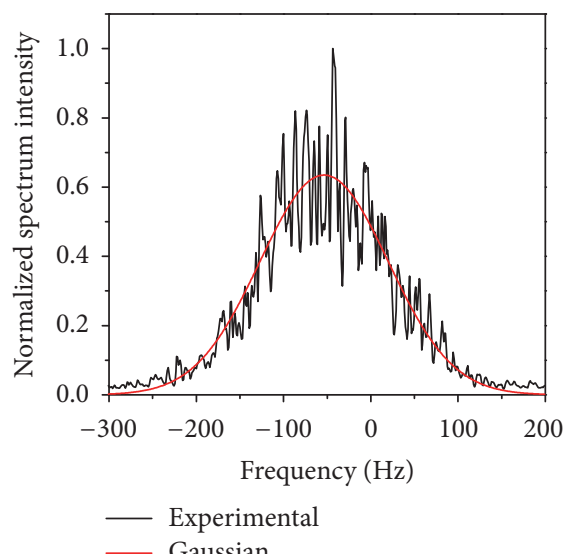

(d)

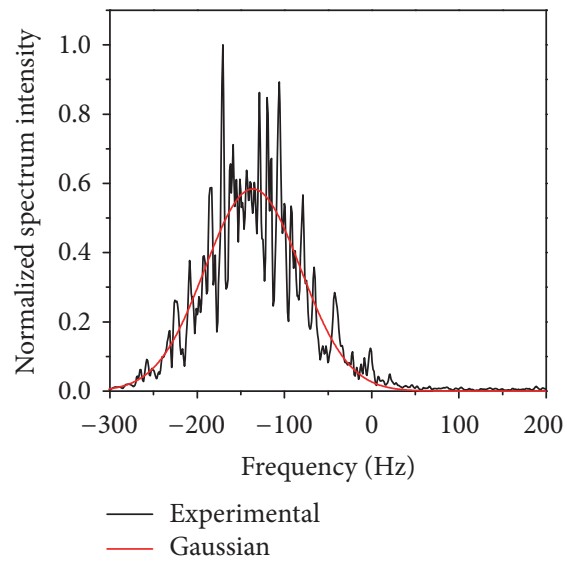

(g)

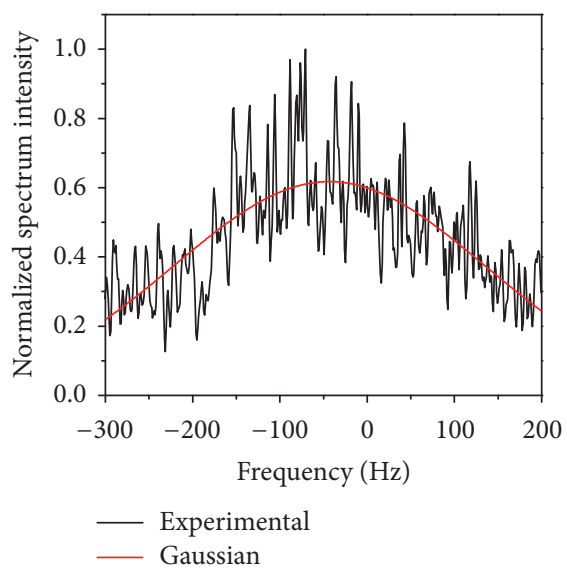

(b)

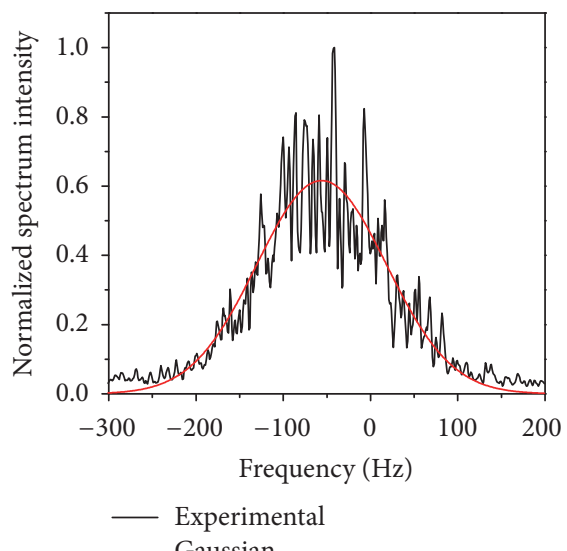

(e)

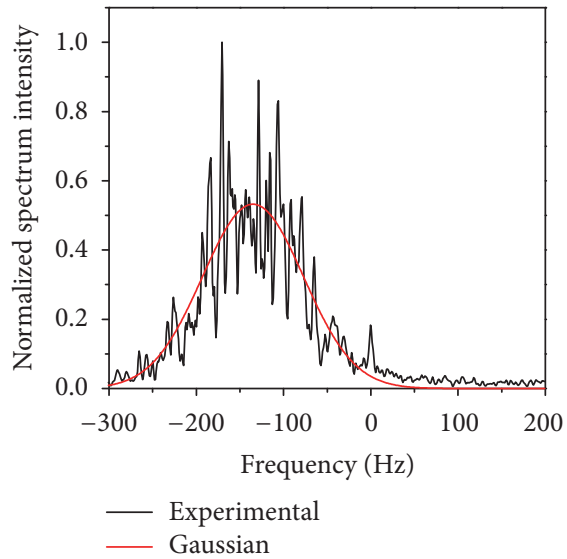

(h)

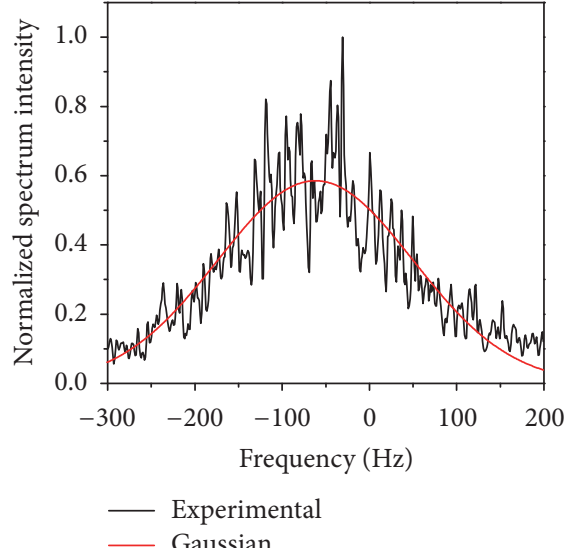

(c)

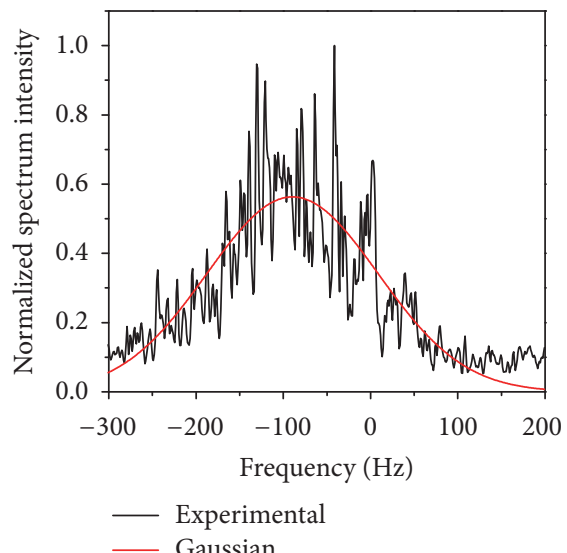

(f)

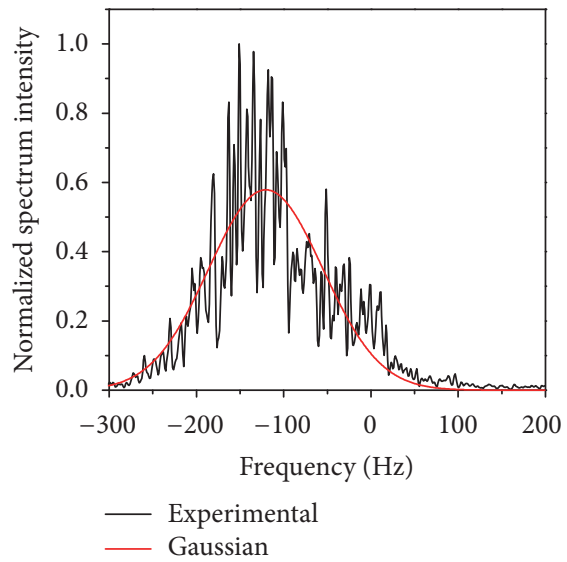

(i)

FIGURE 22: Examples of individual spectra for the different range and time bursts: ((a)-(c)) sum channel and elevation and azimuth difference channels for the 178th range cell within the first time burst. ((d)-(f)) Sum channel and elevation and azimuth difference channels for the 286th range cell within the first time burst. ((g)-(i)) Sum channel and elevation and azimuth difference channels for the 286th range cell within the 45th time burst.

centroid and the width of the Doppler spectra within a time burst for each channel; namely,

$$
\begin{gathered}
m_{f}=P_{1 c} x+P_{2 c}+r_{c} \\
s=P_{1 s} x+P_{2 s}+r_{s},
\end{gathered}
$$

where $r_{c}$ and $r_{s}$ are Gaussian random variables with zero means and standard deviations, $\sigma_{r c}$ and $\sigma_{r s}, P_{1 c}$ and $P_{2 c}$ are the coefficients of the linear model for the centroid of the Doppler spectra, and $P_{1 s}$ and $P_{2 s}$ are those for the spectrum width. As expected, the results show that the linear fits are different between the two channels. This result agrees well 

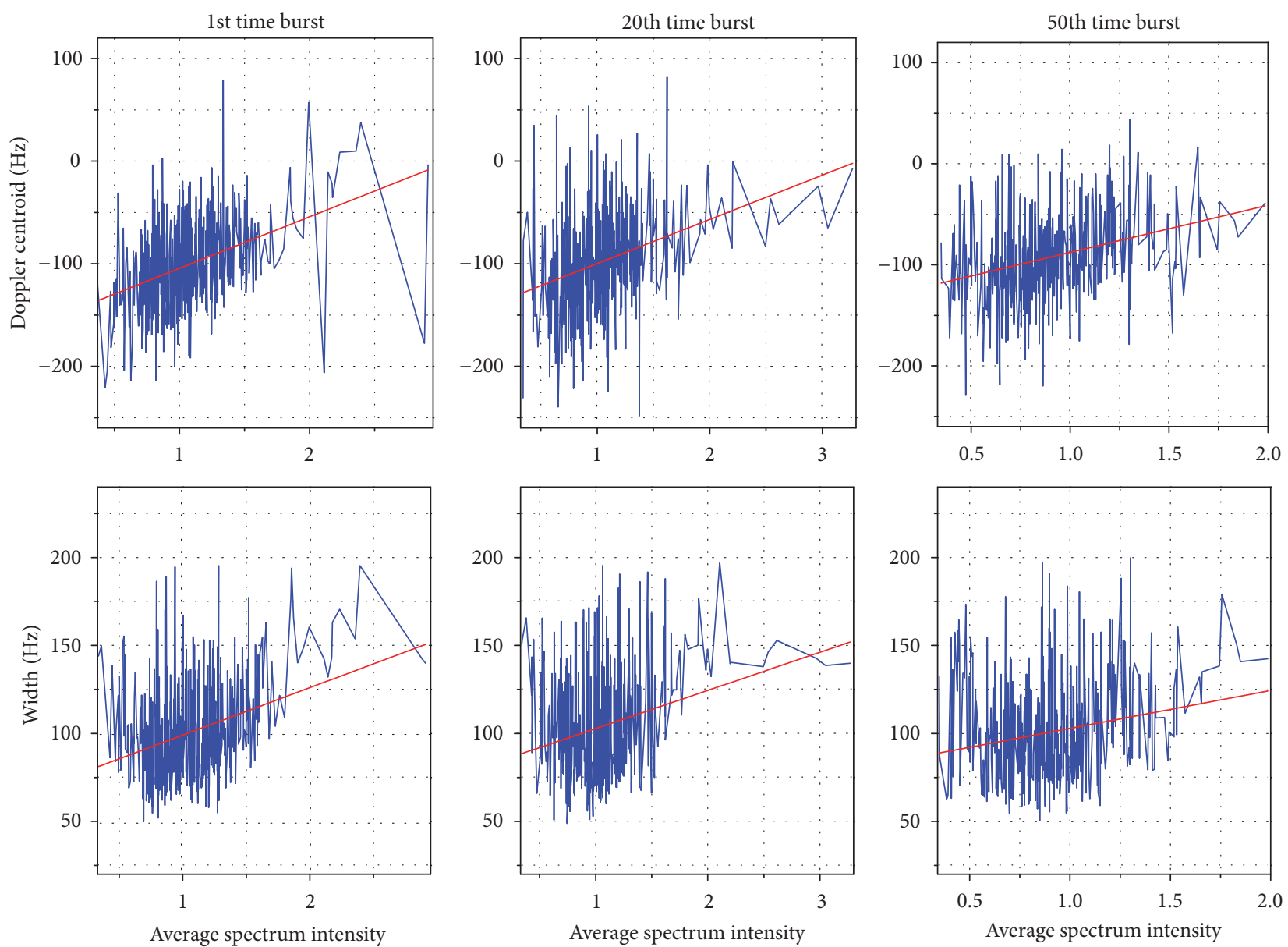

FIGURE 23: Spectra parameters versus intensity for the sum channel, with least-mean-square error straight-line fit: blue, data; red, model fit.

with the results in Figure 21. Note that these linear fits are not constant over entire time bursts for the same channel due to the time-varying nature of the spectra. To further analyze this behavior, a similar process is applied again; that is, the coefficients of the linear models are plotted as functions of the mean of all average spectrum intensities of each time burst. The processing results are shown in Figure 25 for the sum and azimuth difference channels.

From this figure, we observe that there appears to be an evident correlation between the coefficients of the linear models and the mean of all average spectrum intensities of each time burst. Similarly, the fit results indicate that a similar linear model can also be applicable for the prediction of the linear model coefficients (i.e., $P_{1}$ and $P_{2}$ in (17)) of both the centroid and the width of the Doppler spectra. Summarizing the above analyses, it is possible to conclude that the Doppler centroid and the spectrum width can be fundamentally represented by applying the underlying sea clutter intensity for the two-stage linear predictions. The Doppler spectrum can then be reproduced by substituting these predicted Doppler centroids and the spectrum widths into the Gaussian-shaped model. Therefore, this modeling method can be applied for the simulation of successive coherent Doppler spectra. As an example, Figure 26 shows the simulated Doppler PSDs with model parameters taken from the measured sea clutter by exploiting this modeling method. Qualitatively, although there are some differences, these spectra have similar general properties as those in Figures 20 and 21. Again, it is particularly evident that the Doppler centroid and the width of the power spectra fluctuate over range and time. Additionally, in Figure 25, the fit results of the linear model coefficients of the centroid and width of the Doppler spectra are different between the azimuth difference and sum channels. This means that at least two different sets of coefficients are required to represent the linear models of the parameters of the clutter Doppler spectra for all channels of the monopulse radar.

\section{Conclusions and Discussions}

When radar is designed for maritime remote sensing and surveillance, knowledge of sea clutter properties is of great importance for the radar engineer. The main contribution of this paper is providing comprehensive knowledge of the behavior of wideband monopulse clutter by presenting a comparative analysis of the datasets of Ka-band highresolution coherent monopulse radar sea clutter. The lack of studies covering this particular sea clutter data serves as the motivation for addressing this topic. 

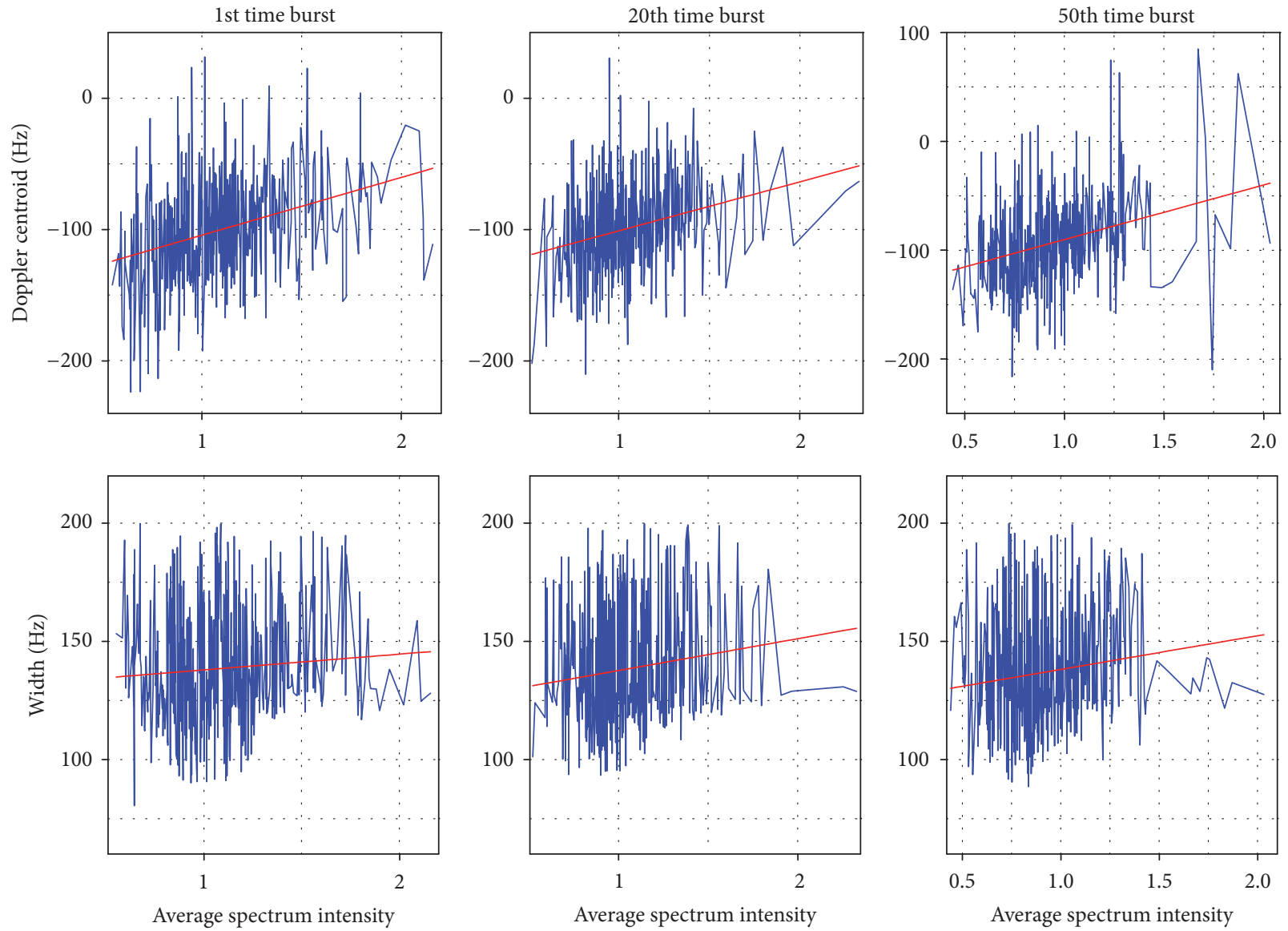

FIGURE 24: Spectra parameters versus intensity for the azimuth difference channel, with least-mean-square error straight-line fit: blue, data; red, model fit.
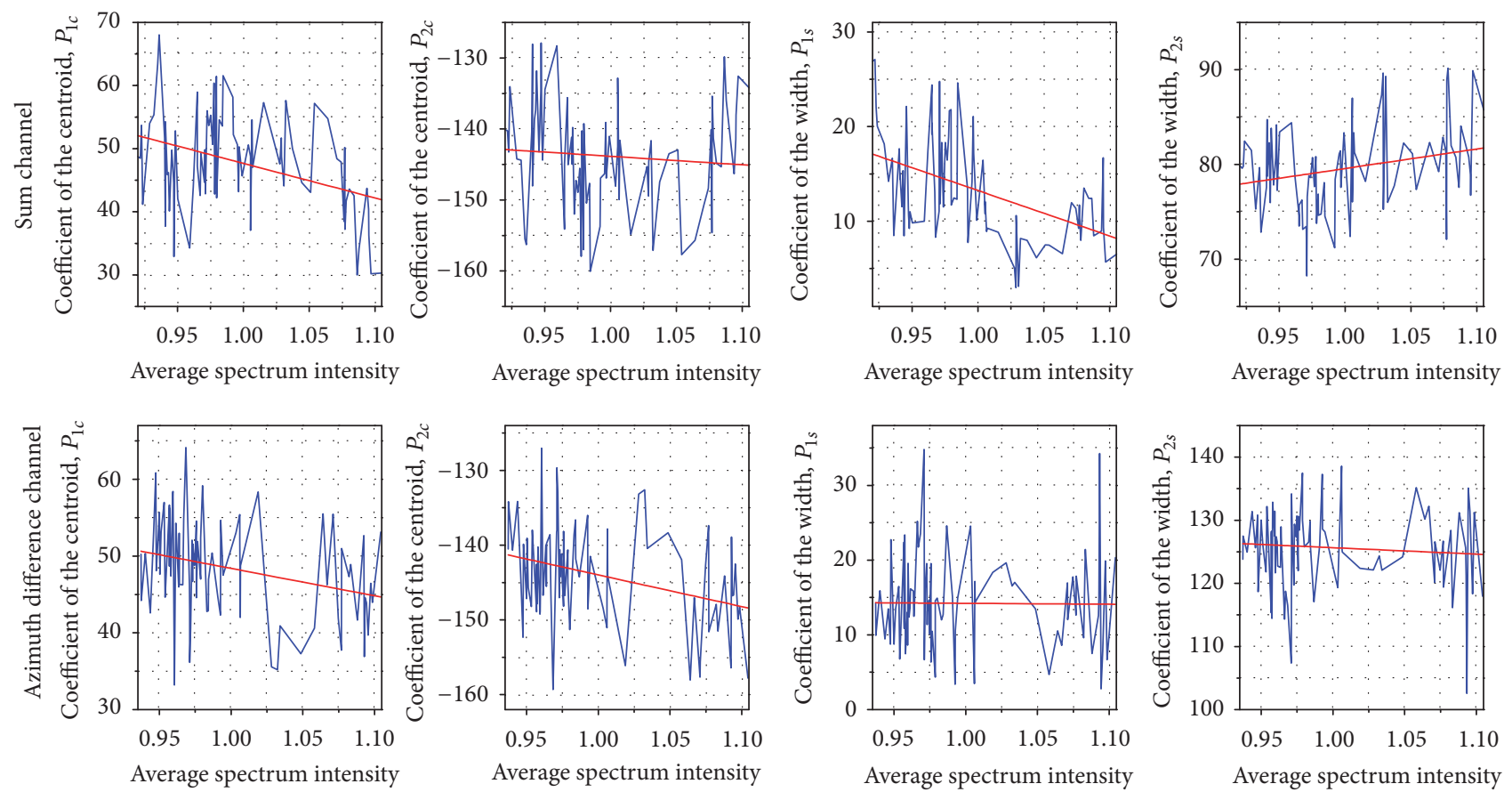

FIGURE 25: The coefficients of the linear models versus intensity for the sum and azimuth difference channels, with least-mean-square error straight-line fit: blue, data; red, model fit. 


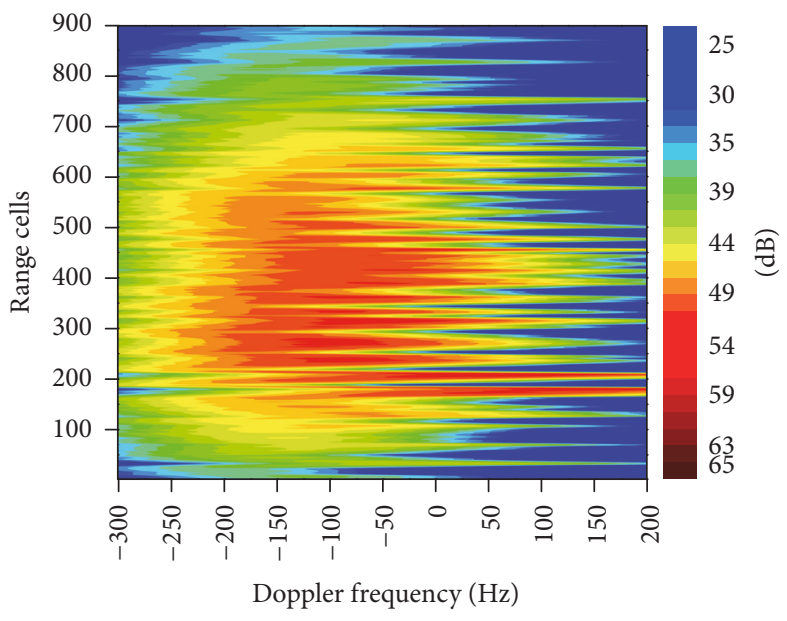

(a)

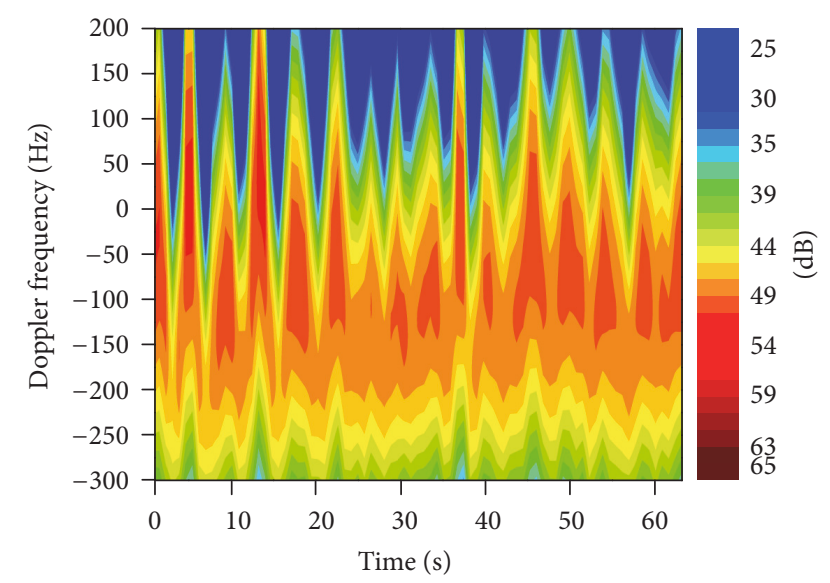

(b)

Figure 26: Examples of the simulated Doppler PSDs for the sum channel: the linear prediction coefficients for $P_{1 c}, P_{2 c}, P_{1 s}$, and $P_{2 s}$ are $\{-55,102\},\{-11,-132\},\{-31,54\}$, and $\{20,59\}$, respectively; $\sigma_{r c}=8 \mathrm{~Hz}, \sigma_{r s}=10 \mathrm{~Hz}^{2}:$ (a) range Doppler maps, the first time burst. (b) Time Doppler maps, the 202nd range cell.

The amplitude statistics of the recorded clutter data were studied first. Four different distributions, namely, the Weibull, $K$, Pareto, and compound inverse Gaussian (CIG) distributions, were applied to fit the clutter observed amplitudes. The fitted results revealed that each of the $K$, Pareto, and CIG distributions can simultaneously provide good fits to the data in most range cells for the three channels of monopulse radar. Considering the widespread applications of the $K$ distribution, the variation of its model parameters with range was investigated further. The results of the estimated parameters show that the scale parameter of the $K$ distribution is closely associated with the clutter powers, which are dominated by the transmitter and receiver antenna gains of the respective channels, and the shape parameter is influenced by the sea state. Moreover, the results indicate that, in the homogeneous sea surface, the $K$ distribution clutter shape parameters of the three channels in each range cell may vary with their respective illumination sea area when the noise is considered.

Additional information about the high-resolution monopulse radar sea clutter was obtained by analyzing the correlation characteristics. Both temporal and spatial analyses were performed for the texture and speckle components of clutter. The results of the correlation analysis suggest that both the temporal and spatial correlation functions of the speckle component are almost unchanged. Moreover, for any one of the temporal and spatial correlation functions, the speckle components of the three channels exhibit similar behavior. Conversely, the temporal correlation function of the texture shows range-varying behavior, and the spatial correlation function also shows time-varying behavior. Additionally, for both the individual temporal and spatial correlations of the texture component, there are apparently similar variations between the elevation difference and sum channels, but there are some differences between the azimuth difference and sum channels. Nonetheless, the averaged results of the correlation coefficients and of the power spectra of the correlation functions exhibit similar behaviors for the three channels and for the two clutter datasets. Meanwhile, regarding the cross-correlations between the sum and difference channels, based on our results, we can conclude that, for the high-resolution radar, both the texture and speckle components are almost completely correlated in elevation, but, in azimuth, the texture component is partly correlated and the speckle component is lowly correlated.

Special attention has been focused on the spectral analysis. The time-varying and range-varying natures of the spectra are very evident for our datasets. For all channels, the individual spectra can be represented by a Gaussianshaped power spectral density with a changing Doppler centroid and a fluctuating spectrum width. Moreover, the spectrum form in the sum channel is similar to that in elevation difference channel but different from that in the azimuth difference channel. Further analysis suggests that the Doppler centroid and the width of the local spectra show a distinct correlation with local spectrum intensity. The modeling method presented by Guan et al. in [35] has been extended here to capture the time-varying and range-varying natures of the Doppler centroid and the width of the local spectra. First, they can be modeled as two linear functions of the mean spectrum intensity along range within a short time interval. The coefficients of the linear models are also correlated with the overall mean of the average spectrum intensities of each time burst. Similarly, the coefficients of the linear models can then be modeled as linear functions of the overall mean of the average spectrum intensities along time.

In summary, the analyses presented in this paper improve the understanding of high-resolution coherent monopulse radar sea clutter. However, more advanced analyses of these data are still necessary in future works. The statistical and spectral properties of the sea clutter spikes, which are helpful for the radar detector in distinguishing between sea clutter 
and true targets, may be studied in a similar way to [17-19], and a detailed analysis about the average radar cross section of sea clutter returns will be presented [20, 21]. Moreover, according to the above analysis results of the statistical properties of wideband monopulse clutter, it will be interesting to study the performance of the detection algorithms using both three-channel return signals, the effects of sea clutter on monopulse angle estimation, and the simulation of highresolution coherent monopulse radar sea clutter data. In addition, sea clutter presents various statistical characteristics depending on the different environmental conditions. We will try our best to design more measurements to collect the sea clutter datasets in various environmental conditions for analyzing.

\section{Conflicts of Interest}

The authors declare that they have no conflicts of interest.

\section{Acknowledgments}

The authors would like to express their sincere thanks to all members of the trail team. It was impossible to collect the trail data without their support.

\section{References}

[1] K. Ward, R. Tough, and S. Watts, Sea Clutter: Scattering, the $K$ Distribution and Radar Performance, IET, London, UK, 2nd edition, 2013.

[2] S. M. Sherman and D. K. Barton, Monopulse Principles and Techniques, Artech House, Norwood, Mass, USA, 2nd edition, 2011.

[3] M. Soumekh, "Moving target detection and imaging using an $\mathrm{X}$ band along-track monopulse SAR," IEEE Transactions on Aerospace and Electronic Systems, vol. 38, no. 1, pp. 315-333, 2002.

[4] M. Rüegg, E. Meier, and D. Nüesch, "Capabilities of dualfrequency millimeter wave SAR with monopulse processing for ground moving target indication," IEEE Transactions on Geoscience and Remote Sensing, vol. 45, no. 3, pp. 539-553, 2007.

[5] D. Wu, D. Zhu, M. Shen, and Y. Li, "Statistical analysis of monopulse-synthetic aperture radar for constant false-alarm rate detection of ground moving targets," IET Radar, Sonar and Navigation, vol. 9, no. 6, pp. 641-652, 2015.

[6] H. Xu, G. Qin, and L. Zhang, "Monopulse radar 3-d imaging and application in terminal guidance radar," in MIPPR 2007: Automatic Target Recognition and Image Analysis; and Multispectral Image Acquisition, vol. 6786 of Proceedings of SPIE, Wuhan, China, November 2007.

[7] V. Anastassopoulos, G. A. Lampropoulos, A. Drosopoulos, and M. Rey, "High resolution radar clutter statistics," IEEE Transactions on Aerospace and Electronic Systems, vol. 35, no. 1, pp. 43-60, 1999.

[8] A. Farina, F. Gini, M. V. Greco, and L. Verrazzani, "High resolution sea clutter data: statistical analysis of recorded live data," IEE Proceedings-Radar, Sonar and Navigation, vol. 144, no. 3, pp. 121-130, 1997.

[9] E. Conte, A. De Maio, and C. Galdi, "Statistical analysis of real clutter at different range resolutions," IEEE Transactions on
Aerospace and Electronic Systems, vol. 40, no. 3, pp. 903-918, 2004.

[10] M. Greco, F. Gini, and M. Rangaswamy, "Statistical analysis of measured polarimetric clutter data at different range resolutions," IEE Proceedings: Radar, Sonar and Navigation, vol. 153, no. 6, pp. 473-481, 2006.

[11] J. Carretero-Moya, J. Gismero-Menoyo, Á. Blanco-Del-campo, and A. Asensio-López, "Statistical analysis of a high-resolution sea-clutter database," IEEE Transactions on Geoscience and Remote Sensing, vol. 48, no. 4, pp. 2024-2037, 2010.

[12] Y. Dong and D. Merrett, "Analysis of L-band multi-channel sea clutter," IET Radar, Sonar and Navigation, vol. 4, no. 2, pp. 223 238, 2010.

[13] W. A. Al-Ashwal, K. Woodbridge, and H. D. Griffiths, "Analysis of bistatic sea clutter-Part II: amplitude statistics," IEEE Transactions on Aerospace and Electronic Systems, vol. 50, no. 2, pp. 1293-1303, 2014

[14] R. Palama, M. S. Greco, P. Stinco, and F. Gini, "Statistical analysis of bistatic and monostatic sea clutter," IEEE Transactions on Aerospace and Electronic Systems, vol. 51, no. 4, pp. 3036-3054, 2015.

[15] A. Fiche, S. Angelliaume, L. Rosenberg, and A. Khenchaf, "Analysis of X-band SAR sea-clutter distributions at different grazing angles," IEEE Transactions on Geoscience and Remote Sensing, vol. 53, no. 8, pp. 4650-4660, 2015.

[16] F. Fioranelli, M. Ritchie, H. Griffiths, S. Sandenbergh, and M. Inggs, "Analysis of polarimetric bistatic sea clutter using the NetRAD radar system," IET Radar, Sonar and Navigation, vol. 10, no. 8, pp. 1356-1366, 2016.

[17] L. Rosenberg, D. J. Crisp, and N. J. Stacy, "Analysis of the KKdistribution with medium grazing angle sea-clutter," IET Radar, Sonar and Navigation, vol. 4, no. 2, pp. 209-222, 2010.

[18] L. Rosenberg, "Sea-spike detection in high grazing angle Xband sea-clutter," IEEE Transactions on Geoscience and Remote Sensing, vol. 51, no. 8, pp. 4556-4562, 2013.

[19] M. Greco, P. Stinco, and F. Gini, "Identification and analysis of sea radar clutter spikes," IET Radar, Sonar and Navigation, vol. 4, no. 2, pp. 239-250, 2010.

[20] W. A. Al-Ashwal, K. Woodbridge, and H. D. Griffiths, "Analysis of bistatic sea clutter-part I: average reflectivity," IEEE Transactions on Aerospace and Electronic Systems, vol. 50, no. 2, pp. 1283-1292, 2014.

[21] H. D. Griffiths, W. A. Al-Ashwal, K. D. Ward, R. J. A. Tough, C. J. Baker, and K. Woodbridge, "Measurement and modelling of bistatic radar sea clutter," IET Radar, Sonar and Navigation, vol. 4, no. 2, pp. 280-292, 2010.

[22] M. Sekine and Y. H. Mao, Weibull Radar Clutter, Peter Peregrinus, London, UK, 1990.

[23] V. Anastassopoulos and G. A. Lampropoulos, "Optimal CFAR detection in weibull clutter," IEEE Transactions on Aerospace and Electronic Systems, vol. 31, no. 1, pp. 52-64, 1995.

[24] S. Watts, "Radar detection prediction in K-distributed sea clutter and thermal noise," IEEE Transactions on Aerospace and Electronic Systems, vol. 23, no. 1, pp. 40-45, 1987.

[25] L. Rosenberg and S. Bocquet, "The Pareto distribution for high grazing angle sea-clutter," in Proceedings of the 33rd IEEE International Geoscience and Remote Sensing Symposium (IGARSS '13), pp. 4209-4212, IEEE, July 2013.

[26] A. Balleri, A. Nehorai, and J. Wang, "Maximum likelihood estimation for compound-Gaussian clutter with inverse gamma texture," IEEE Transactions on Aerospace and Electronic Systems, vol. 43, no. 2, pp. 775-780, 2007. 
[27] G. V. Weinberg, "Assessing Pareto fit to high-resolution highgrazing-angle sea clutter," Electronics Letters, vol. 47, no. 8, pp. 516-517, 2011.

[28] E. Ollila, D. E. Tyler, V. Koivunen, and H. V. Poor, "CompoundGaussian clutter modeling with an inverse gaussian texture distribution," IEEE Signal Processing Letters, vol. 19, no. 12, pp. 876-879, 2012.

[29] A. Mezache, F. Soltani, M. Sahed, and I. Chalabi, "Model for non-rayleigh clutter amplitudes using compound inverse gaussian distribution: an experimental analysis," IEEE Transactions on Aerospace and Electronic Systems, vol. 51, no. 1, pp. 142-153, 2015.

[30] D. J. Crisp, L. Rosenberg, N. J. Stacy, and Y. Dong, "Modelling $\mathrm{X}$-band sea clutter with the K-distribution: shape parameter variation," in Proceedings of the International Radar Conference "Surveillance for a Safer World" (RADAR '09), pp. 1-6, December 2009.

[31] S. Watts, K. Ward, and R. Tough, "Modelling the shape parameter of sea clutter," in Proceedings of the International Radar Conference-Surveillance for a Safer World (RADAR '09), pp. 16, 2009.

[32] Y. Yang, S.-P. Xiao, D.-J. Feng, and W.-M. Zhang, "Modelling and simulation of spatial-temporal correlated $\mathrm{K}$ distributed clutter for coherent radar seeker," IET Radar, Sonar and Navigation, vol. 8, no. 1, pp. 1-8, 2014.

[33] J. Wang and $\mathrm{X} . \mathrm{Xu}$, "Simulation of correlated low-grazingangle sea clutter based on phase retrieval," IEEE Transactions on Geoscience and Remote Sensing, vol. 53, no. 7, pp. 3917-3930, 2015.

[34] Y. Dong, "High grazing angle and high resolution sea clutter: correlation and polarisation analyses," Research Report DSTOTR-1972, 2007.

[35] J. Guan, H. Ding, Y. Huang, G. Wang, and Y. He, "Spatial correlation property with measured sea clutter data," Chinese Journal of Radio Science, vol. 27, no. 5, pp. 943-953, 2012.

[36] H. Ding, J. Guan, N. Liu, and G. Wang, "New spatial correlation models for sea clutter," IEEE Geoscience and Remote Sensing Letters, vol. 12, no. 9, pp. 1833-1837, 2015.

[37] S. Watts, "Modeling and simulation of coherent sea clutter," IEEE Transactions on Aerospace and Electronic Systems, vol. 48, no. 4, pp. 3303-3317, 2012.

[38] S. Watts, L. Rosenberg, S. Bocquet, and M. Ritchie, "Doppler spectra of medium grazing angle sea clutter; part 1: characterisation," IET Radar, Sonar and Navigation, vol. 10, no. 1, pp. 24-31, 2016.

[39] S. Bocquet, "Parameter estimation for Pareto and K distributed clutter with noise," IET Radar, Sonar and Navigation, vol. 9, no. 1, pp. 104-113, 2015.

[40] I. R. Joughin, D. B. Percival, and D. P. Winebrenner, "Maximum likelihood estimation of $\mathrm{K}$ distribution parameters for SAR data," IEEE Transactions on Geoscience and Remote Sensing, vol. 31, no. 5, pp. 989-999, 1993.

[41] D. A. Abraham and A. P. Lyons, "Reliable methods for estimating the K-distribution shape parameter," IEEE Journal of Oceanic Engineering, vol. 35, no. 2, pp. 288-302, 2010.

[42] S. Bocquet, "Closed-form parameter estimators for Pareto distributed clutter with noise," Electronics Letters, vol. 51, no. 23, pp. 1924-1926, 2015.

[43] A. Mezache, M. Sahed, T. Laroussi, and D. Chikouche, "Two novel methods for estimating the compound K-clutter parameters in presence of thermal noise," IET Radar, Sonar and Navigation, vol. 5, no. 9, pp. 934-942, 2011.
[44] J. C. Lagarias, J. A. Reeds, M. Wright, and P. E. Wright, "Convergence properties of the Nelder-Mead simplex method in low dimensions," SIAM Journal on Optimization, vol. 9, no. 1, pp. 112-147, 2006.

[45] D. Middleton, "New physical-statistical methods and models for clutter and reverberation: the kA-distribution and related probability structures," IEEE Journal of Oceanic Engineering, vol. 24, no. 3, pp. 261-284, 1999.

[46] Y. Dong, "Distribution of x-band high resolution and high grazing angle sea clutter," Research Report DSTO-RR-0316, 2006.

[47] E. Conte, M. Di Bisceglie, C. Galdi, and G. Ricci, "A procedure for measuring the coherence length of the sea texture," IEEE Transactions on Instrumentation and Measurement, vol. 46, no. 4, pp. 836-841, 1997.

[48] F. Gini and M. Greco, "Texture modelling, estimation and validation using measured sea clutter data," IEE Proceedings: Radar, Sonar and Navigation, vol. 149, no. 3, pp. 115-124, 2002.

[49] K. Ing, M. R. Morelande, S. Suvorova, and B. Moran, "Parametric texture estimation and prediction using measured sea clutter data," IET Radar, Sonar and Navigation, vol. 10, no. 3, pp. 449458, 2016.

[50] M. Greco, F. Bordoni, and F. Gini, "X-band sea-clutter nonstationarity: influence of long waves," IEEE Journal of Oceanic Engineering, vol. 29, no. 2, pp. 269-283, 2004.

[51] E. Conte and M. Longo, "Characterisation of radar clutter as a spherically invariant random process," IEE Proceedings F: Communications, Radar and Signal Processing, vol. 134, no. 2, pp. 191-197, 1987.

[52] S. Haykin and D. J. Thomson, "Signal detection in a nonstationary environment reformulated as an adaptive pattern classification problem," Proceedings of the IEEE, vol. 86, no. 11, pp. 2325-2344, 1998.

[53] Y.-L. Shi and P.-L. Shui, "Target detection in high-resolution sea clutter via block-adaptive clutter suppression," IET Radar, Sonar and Navigation, vol. 5, no. 1, pp. 48-57, 2011.

[54] D.-C. Li and P.-L. Shui, "Floating small target detection in sea clutter via normalised Doppler power spectrum," IET Radar, Sonar and Navigation, vol. 10, no. 4, pp. 699-706, 2016.

[55] R. D. Brown, R. A. Schneible, M. C. Wicks, H. Wang, and Y. Zhang, "STAP for clutter suppression with sum and difference beams," IEEE Transactions on Aerospace and Electronic Systems, vol. 36, no. 2, pp. 634-646, 2000.

[56] L. Zhao, J. Sun, and X. Xu, "A hybrid STAP approach to target detection for heterogeneous scenarios in radar seekers," Multidimensional Systems and Signal Processing, vol. 25, no. 3, pp. 493-509, 2014.

[57] L. Rosenberg, "Characterization of high grazing angle X-band sea-clutter doppler spectra," IEEE Transactions on Aerospace and Electronic Systems, vol. 50, no. 1, pp. 406-417, 2014.

[58] A. Farina, F. Gini, M. V. Greco, and P. H. Y. Lee, "Improvement factor for real sea clutter doppler frequency spectra," IEE Proceedings-Radar, Sonar and Navigation, vol. 143, no. 5, pp. 341-344, 1996. 


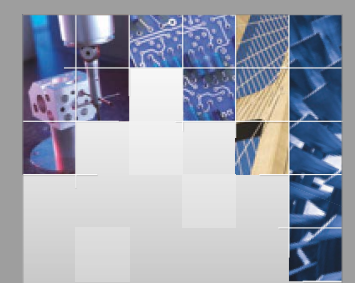

\section{Enfincering}
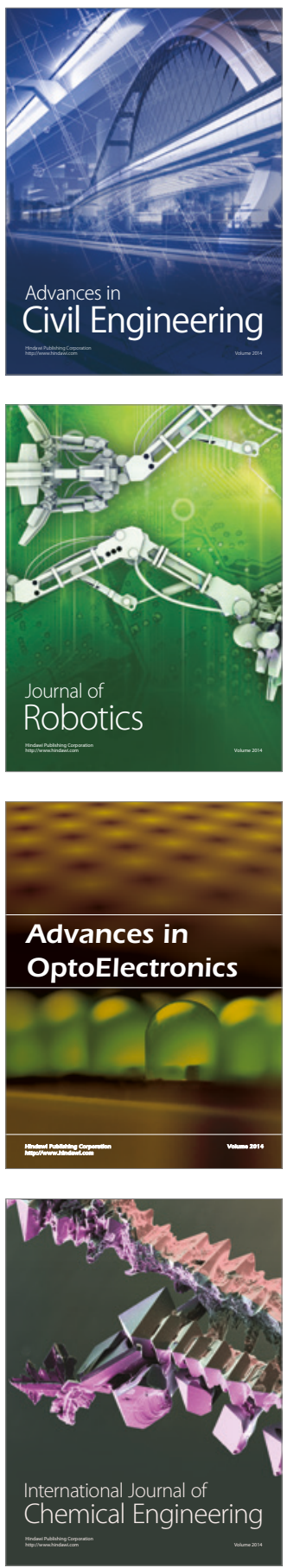

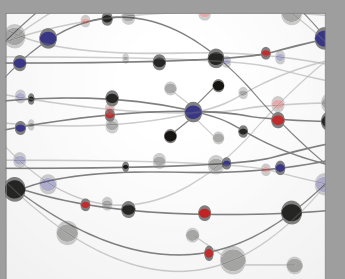

The Scientific World Journal

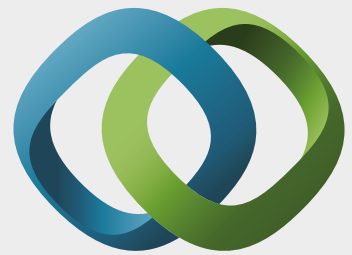

\section{Hindawi}

Submit your manuscripts at

https://www.hindawi.com
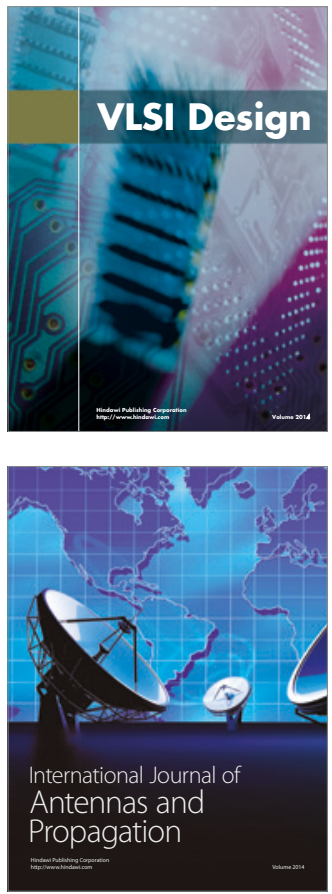

\section{Rotating}

Machinery
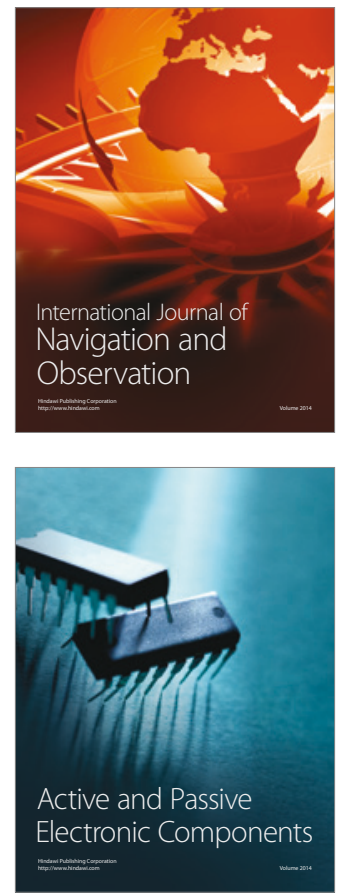
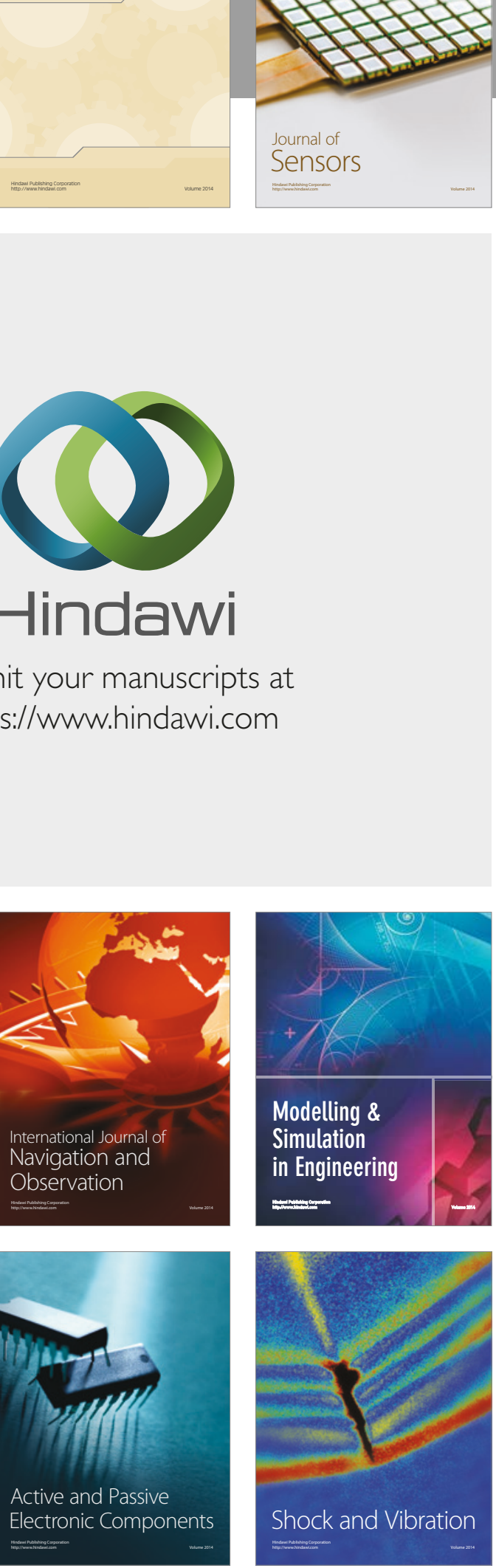
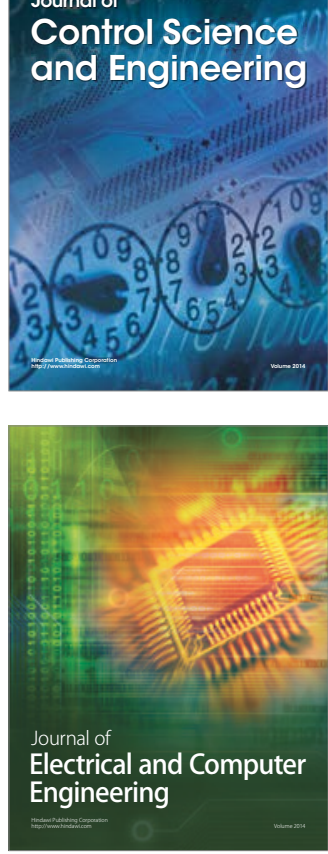

Distributed

Journal of

Control Science

and Engineering
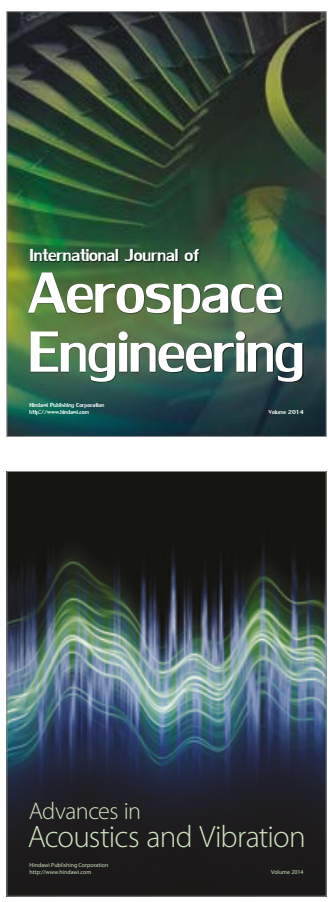

Sensor Networks 